Supporting Information for

\title{
Controlled Ring-Opening Polymerization of $\beta$-Butyrolactone via Bifunctional Organoboron Catalysts
}

Li Yang $\$$ Yao-Yao Zhang $\$$, Guan-Wen Yang, Rui Xie, and Guang-Peng Wu*

Dedicated to Professor Donald J. Darensbourg on the occasion of his 80th birthday.

MOE Laboratory of Macromolecular Synthesis and Functionalization, Key laboratory of Adsorption and Separation Materials and Technologies of Zhejiang Province, Department of Polymer Science and Engineering, Zhejiang University, Hangzhou 310027, China 


\section{TABLE OF CONTENTS}

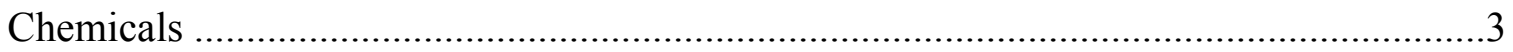

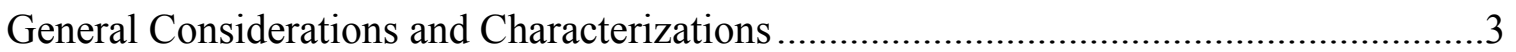

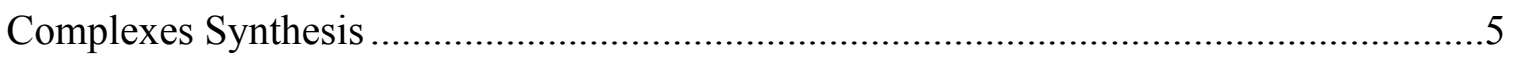

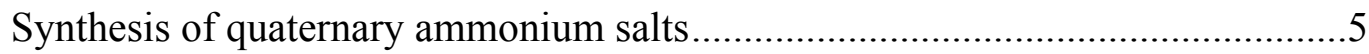

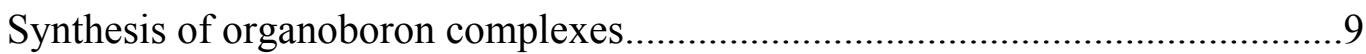

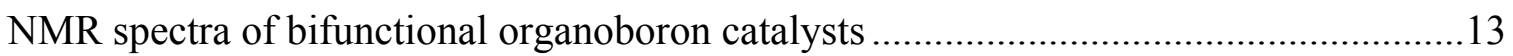

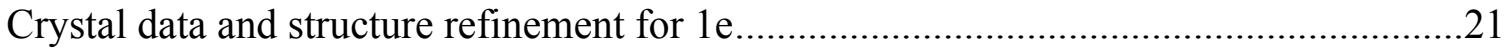

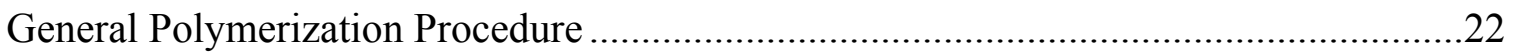

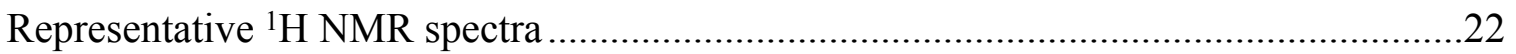

Representative DSC curves of atactic and isotactic PHBs ............................................24

The determination of carboxylate end-group by diphenylchlorophosphate $\left((\mathrm{PhO})_{2} \mathrm{POCl}\right)$.

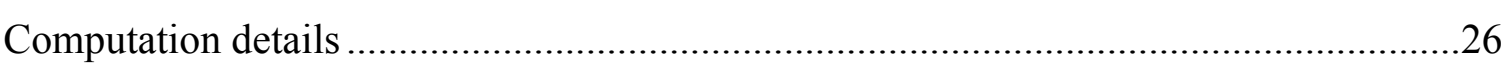

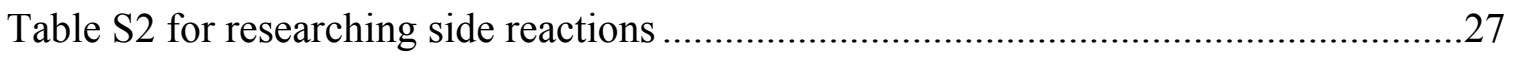

Table S3 for investigating the importance of quaternary ammonium and boron moiety. .28

The detailed MALDI-TOF spectrum of PHB in Figure 4A ………….........................28

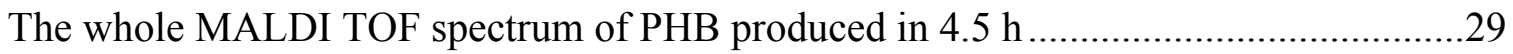

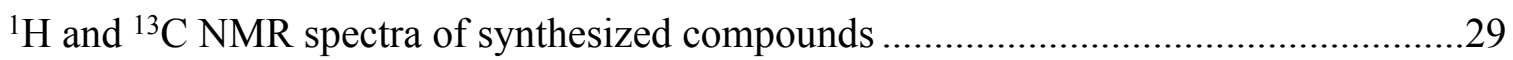

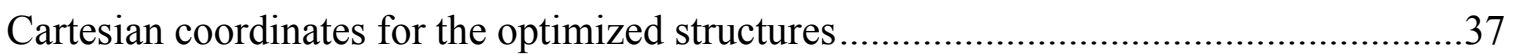

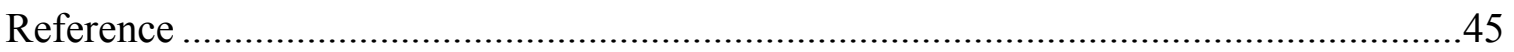




\section{Chemicals}

Tetrahydrofuran (THF), toluene, $N, N$-dimethylformamide (DMF), $n$-hexane, and dichloromethane were purchased from Sinopharm Chemical Reagent Co., Ltd. Calcium hydride $\left(\mathrm{CaH}_{2}\right)$ were purchased from Meryer Co. Deuterated solvents were purchased from Cambridge Isotope Laboratories. Toluene, $n$-hexane, and THF were distilled over sodium/benzophenone under nitrogen. DMF was dried over $\mathrm{CaH}_{2}$ overnight, followed by vacuum distillation. $\mathrm{CDCl}_{3}$ was distilled over $\mathrm{CaH}_{2}$ for $12 \mathrm{~h}$ and stored over activated Davison $4 \AA$ molecular sieves. Racemic $\beta$-butyrolactone (rac-BBL) was purchased from Meryer Co., and dried with $\mathrm{CaH}_{2}$ for 3 days, then freshly distilled under reduced

pressure, and eventually degassed thoroughly by freeze-pump-thaw cycles prior to use. Notation: BBL is a suspected carcinogen, thus, it should be handled with appropriate safeguards. Sodium benzoate was purchased from Sinopharm Chemical Reagent Co., and dehydration at $120{ }^{\circ} \mathrm{C}$ in vacuum. All the other raw materials for catalysts preparation in this work including 9-borabicyclo[3.3.1]nonane (9-BBN), halogenated hydrocarbons and amines were purchased from Energy Chemical Co. and Meryer Co. and used without further purification. Amberlite(R) IRA-400 (Cl) resin was received from Alfa Aesar Chemical Co. and washed with methanol before usage.

\section{General Considerations and Characterizations}

All reactions and manipulations with air- and/or moisture-sensitive compounds were carried out in glovebox filled with dry nitrogen or using a standard dual-manifold Schlenk line techniques.

Nuclear NMR spectra were recorded on a Bruker AVANCE III 400 spectrometer $\left({ }^{1} \mathrm{H}\right.$ NMR $400 \mathrm{MHz},{ }^{13} \mathrm{C}$ NMR $101 \mathrm{MHz},{ }^{31} \mathrm{P}$ NMR 162MHz), a Bruker AVANCE III 500 spectrometer $\left({ }^{1} \mathrm{H}\right.$ NMR $500 \mathrm{MHz},{ }^{13} \mathrm{C}$ NMR $\left.126 \mathrm{MHz}\right)$ at $25{ }^{\circ} \mathrm{C}$ and referenced the internal or external standard shift or the residual solvent chemical shifts $\left({ }^{1} \mathrm{H}\right.$ : TMS in 
$\mathrm{CDCl}_{3}=0$ ppm, ${ }^{13} \mathrm{C}: \mathrm{CH}_{3} \mathrm{OH}$ in $\left.\mathrm{CDCl}_{3}=50.41 \mathrm{ppm}, \mathrm{CDCl}_{3}=77.36 \mathrm{ppm}\right)$.

X-ray crystallographic data were collected at $170 \mathrm{~K}$ on a BrukerAXS D8 VENTURE diffractometer equipped with a PHOTON-100/CMOS detector (MoK $\alpha, \lambda=$ $0.71073 \AA$ ). Indexing was performed using APEX2. Employing Olex2, the crystal structure was solved with the ShelXT structure solution program using Intrinsic Phasing and refined with the ShelXT refinement package through Least Squares minimization. All non-hydrogen atoms were refined anisotropically, generated geometrically, and assigned appropriate isotropic thermal parameters. The contribution of disordered solvent molecules was treated as diffuse using the Squeeze routine implemented in Platon.

High resolution mass spectra (HRMS) were conducted on an Agilent 6545 Q-TOF instrument using ESI ionization.

Gel permeation chromatography (GPC) was carried out using a system equipped with a Waters Chromatography, Inc. model 1515 isocratic pump, a model 2414 differential refractometer, and a two Polymer Laboratories, Inc. Styragel columns (PLgel $5 \mu \mathrm{m}$ Mixed C, $500 \AA$, and $104 \AA, 300 \times 7.5 \mathrm{~mm}$ columns) in series. After the system was equilibrated at $35{ }^{\circ} \mathrm{C}$ in THF, the GPC columns were eluted with THF at $35{ }^{\circ} \mathrm{C}$ at 1 $\mathrm{mL} / \mathrm{min}$ and were calibrated by a series of narrowly dispersed polystyrene (PS) standards. Unless stated otherwise, all GPC samples were of the precipitated or purified polymers.

Matrix-assisted laser desorption ionization time-of-flight mass spectrometry (MALDI TOF) analyses were conducted on a Bruker Daltonics UltrafleXtreme system. Purified polymer samples were dissolved in THF at a concentration of $10 \mathrm{mg} / \mathrm{mL}$. 2,5-dihydroxybenzoic acid (DHB) was chosen as the matrix and the resulting spectra were analyzed using the Bruker Daltonics flexAnalysis 3.4 software package.

In situ IR spectroscopic reactions were carried out with a Mettler-Toledo ReactIR 15 Reaction Analysis System fitted with a Sentinel DiComp (diamond) High-Pressure Probe. Data were obtained and analyzed using ReactIR software version 7.0, the infrared 
spectrometer was set to collect one spectrum every $15 \mathrm{~s}$.

Polymer melting points were measured by Differential scanning calorimetry (DSC). DSC measurements were performed on a NETZSCH DSC 214 Polyma instrument (NETZSCH, Germany) equipped with an IC70 intra cooler. Polymer samples were analyzed using the following heating program: $-30{ }^{\circ} \mathrm{C}$ to $200{ }^{\circ} \mathrm{C}$ at $20 \mathrm{~K} / \mathrm{min}, 200{ }^{\circ} \mathrm{C}$ to $-70{ }^{\circ} \mathrm{C}$ at $10 \mathrm{~K} / \mathrm{min}$, and then $-70{ }^{\circ} \mathrm{C}$ to $200{ }^{\circ} \mathrm{C}$ at $20 \mathrm{~K} / \mathrm{min}$. The glass transition temperature $\left(\mathrm{T}_{\mathrm{g}}\right)$ values were taken as the midpoint of the inflection tangent from the second heating cycles, and melting points were taken upon the third heating scan.

\section{Complexes Synthesis}

\section{Synthesis of quaternary ammonium salts}

All the quaternary ammonium salts were synthesized following the analogous procedures reported early and the main operations were simply described as follows. Adding the tertiary amine (1.0 equiv.) and the corresponding equimolar alkenyl halide to a round bottom flask equipped with a stir bar, and employing acetonitrile $(100 \mathrm{~mL})$ as solvent. After the clear solution refluxed at $95{ }^{\circ} \mathrm{C}$ for 2 days, it was cooled to room temperature and concentrated in vacuo, followed by further purification with ethyl acetate for three times. Eventually, the obtained solid was isolated by vacuum filtration and dried in vacuum to a constant weight. Additionally, in some circumstances, a step of salt metathesis was needed in order to generate complexes charged with chloride since alkenyl bromide possesses a higher activity than alkenyl chloride in the Menschutkin reaction, however, the solubility of sodium chloride is lower than sodium bromide in dichloromethane so that sodium chloride would be precipitated, giving catalysts with higher purity. Therefore, a salt metathesis of $\mathrm{Br}^{-}$to $\mathrm{Cl}^{-}$is needed. $N, N$, $N$-tripropylpent-4-en-1-aminium bromide, $N, N, N$-tributylpent-4-en-1-aminium bromide 
and complexes 1a, $\mathbf{1 b}$, and $\mathbf{1 c}$ were prepared following the reported procedures. ${ }^{1-3}$

\section{$N$-butyl- $N$, $N$-dimethylbut-3-en-1-aminium bromide}

$\underbrace{\text { Following the general procedures, a }}_{\mathrm{Br}^{\ominus}} \begin{gathered}\text { solution } \\ N, N \text {-dimethylbutan-1-amine }(10.1 \mathrm{~g}, 0.10 \mathrm{~mol}, 1.00 \text { equiv. }) \text { in }\end{gathered}$ acetonitrile was treated with 4-bromobut-1-ene (13.5 g, $0.10 \mathrm{~mol}, 1.00$ equiv.) and potassium carbonate (20.7 g, $0.15 \mathrm{~mol}, 1.50$ equiv.). The product was isolated as a white solid (20.1 g, 85\% yield). $\left.{ }^{1} \mathrm{H} \mathrm{NMR} \mathrm{(500} \mathrm{MHz,} \mathrm{CDCl}_{3}\right): \delta 5.81$ (ddt, $\mathrm{J}=13.3$, 9.5, 6.7 Hz, 1H), $5.24(\mathrm{dd}, \mathrm{J}=50.0,13.7 \mathrm{~Hz}, 2 \mathrm{H}), 3.79-3.57(\mathrm{~m}, 4 \mathrm{H}), 3.42(\mathrm{~s}, 6 \mathrm{H}), 2.60$ $(\mathrm{dd}, \mathrm{J}=15.0,6.8 \mathrm{~Hz}, 2 \mathrm{H}), 1.85-1.66(\mathrm{~m}, 2 \mathrm{H}), 1.46(\mathrm{dq}, \mathrm{J}=14.4,7.2 \mathrm{~Hz}, 2 \mathrm{H}), 1.01(\mathrm{t}, \mathrm{J}$ $=7.2 \mathrm{~Hz}, 3 \mathrm{H}) \cdot{ }^{13} \mathrm{C} \mathrm{NMR}\left(126 \mathrm{MHz}, \mathrm{CDCl}_{3}\right): \delta 131.62,119.55,64.23,62.93,51.37$, $27.54,24.71,19.63,13.79$.

\section{$N$-butyl- $N$, $N$-dimethylbut-3-en-1-aminium chloride}

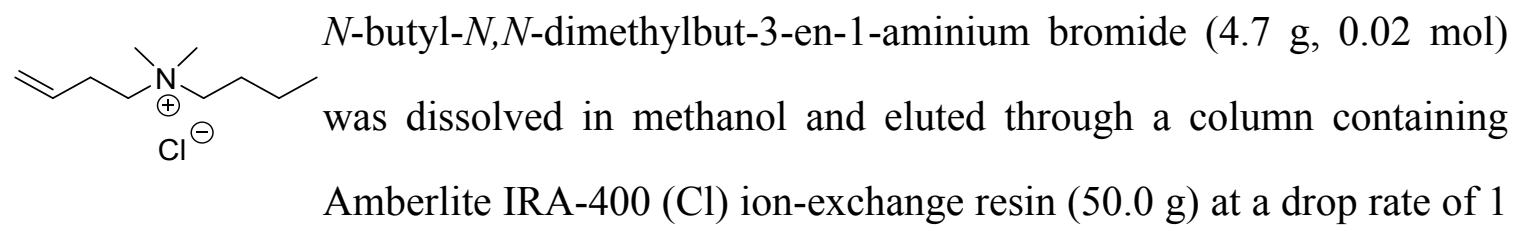

$\mathrm{mL} / \mathrm{min}$. Solvent was removed under reduced pressure at $50{ }^{\circ} \mathrm{C}$, affording a white solid (3.8 g, 98\% yield). ${ }^{1} \mathrm{H}$ NMR $\left(500 \mathrm{MHz}, \mathrm{CDCl}_{3}\right): \delta 5.87-5.70(\mathrm{~m}, 1 \mathrm{H}), 5.33-5.16(\mathrm{~m}$, 2H), $3.70-3.54(\mathrm{~m}, 4 \mathrm{H}), 3.46(\mathrm{~s}, 6 \mathrm{H}), 2.56(\mathrm{dd}, \mathrm{J}=15.7,7.0 \mathrm{~Hz}, 2 \mathrm{H}), 1.76-1.66(\mathrm{~m}$, 2H), $1.49-1.40(\mathrm{~m}, 2 \mathrm{H}), 1.01(\mathrm{t}, \mathrm{J}=7.4 \mathrm{~Hz}, 3 \mathrm{H}) .{ }^{13} \mathrm{C}$ NMR $\left(126 \mathrm{MHz}, \mathrm{CDCl}_{3}\right): \delta$ $131.54,119.69,63.92,62.65,51.40,27.56,24.75,19.71,13.79$.

\section{$N$-butyl- $N, N$-dimethylpent-4-en-1-aminium bromide}

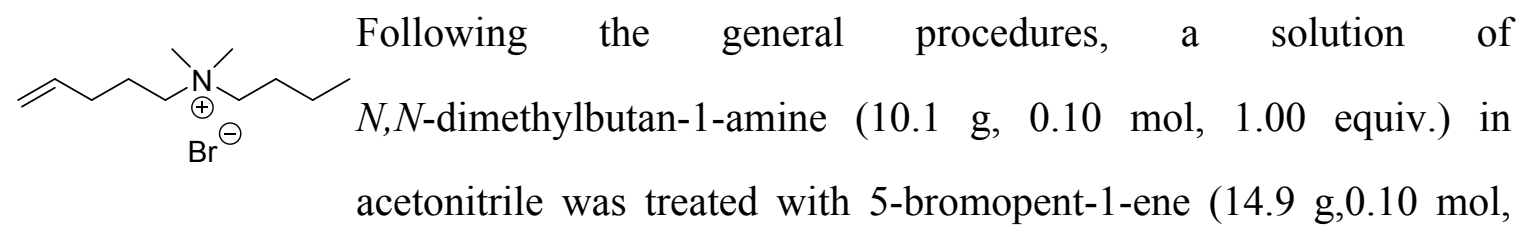

1.00 equiv.). The product was isolated as an off-white solid (24.8 g, 99\% yield). ${ }^{1} \mathrm{H}$ NMR 
$\left(500 \mathrm{MHz}, \mathrm{CDCl}_{3}\right): \delta 5.80(\mathrm{ddt}, \mathrm{J}=16.9,10.2,6.7 \mathrm{~Hz}, 1 \mathrm{H}), 5.18-5.03(\mathrm{~m}, 2 \mathrm{H}), 3.67-$ $3.55(\mathrm{~m}, 4 \mathrm{H}), 3.39(\mathrm{~s}, 6 \mathrm{H}), 2.21(\mathrm{q}, \mathrm{J}=6.9 \mathrm{~Hz}, 2 \mathrm{H}), 1.94-1.85(\mathrm{~m}, 2 \mathrm{H}), 1.78-1.69(\mathrm{~m}$, 2H), $1.52-1.40(\mathrm{~m}, 2 \mathrm{H}), 1.00(\mathrm{t}, \mathrm{J}=7.4 \mathrm{~Hz}, 3 \mathrm{H}) .{ }^{13} \mathrm{C} \mathrm{NMR}\left(126 \mathrm{MHz}, \mathrm{CDCl}_{3}\right): \delta$ $135.42,116.52,63.58,63.05,50.80,29.58,24.21,21.51,19.15,13.32$.

\section{$N$-butyl- $N, N$-dimethylpent-4-en-1-aminium chloride}

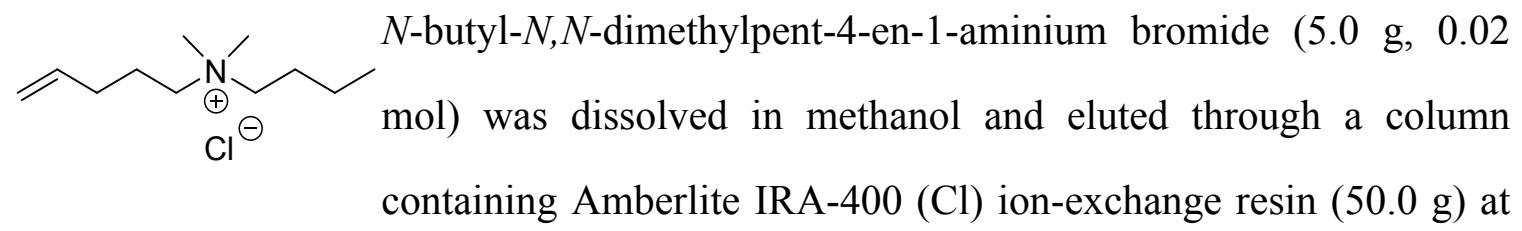

a drop rate of $1 \mathrm{~mL} / \mathrm{min}$. Solvent was removed under reduced pressure at $50{ }^{\circ} \mathrm{C}$, affording an off-white solid (4.1 g, 99\% yield). ${ }^{1} \mathrm{H}$ NMR (500 MHz, $\left.\mathrm{CDCl}_{3}\right): \delta 5.79(\mathrm{ddt}, \mathrm{J}=16.9$, 10.1, $6.7 \mathrm{~Hz}, 1 \mathrm{H}), 5.16-5.02(\mathrm{~m}, 2 \mathrm{H}), 3.63-3.54(\mathrm{~m}, 4 \mathrm{H}), 3.40(\mathrm{~s}, 6 \mathrm{H}), 2.20$ (q, J = 6.9 $\mathrm{Hz}, 2 \mathrm{H}), 1.93-1.81(\mathrm{~m}, 2 \mathrm{H}), 1.79-1.66(\mathrm{~m}, 2 \mathrm{H}), 1.51-1.39(\mathrm{~m}, 2 \mathrm{H}), 1.00(\mathrm{t}, \mathrm{J}=7.3$ $\mathrm{Hz}, 3 \mathrm{H}) .{ }^{13} \mathrm{C} \mathrm{NMR}\left(126 \mathrm{MHz}, \mathrm{CDCl}_{3}\right): \delta 135.65,116.83,63.60,63.07,50.97,29.90$, $24.48,21.77,19.47,13.57$.

\section{$N$-butyl- $N$, $N$-dimethylhex-5-en-1-aminium bromide}

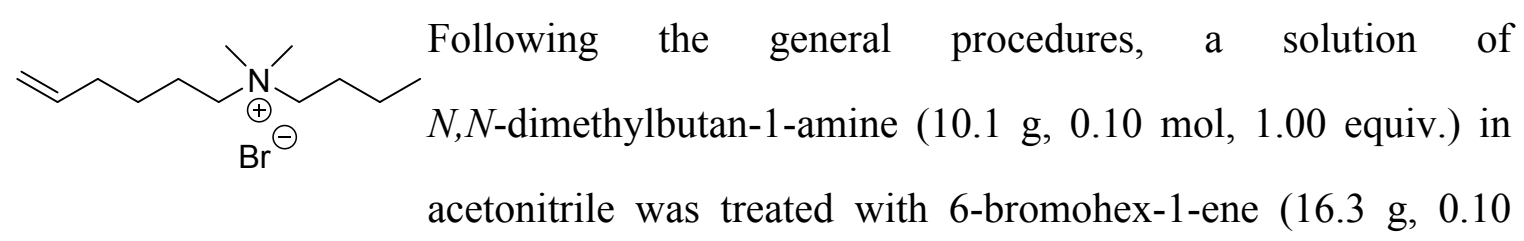

mol, 1.00 equiv.). The product was isolated as a tan solid $(25.4 \mathrm{~g}, 96 \%$ yield $) .{ }^{1} \mathrm{H} \mathrm{NMR}$ $\left(500 \mathrm{MHz}, \mathrm{CDCl}_{3}\right): \delta 5.77(\mathrm{ddt}, \mathrm{J}=13.6,10.2,6.7 \mathrm{~Hz}, 1 \mathrm{H}), 5.02(\mathrm{dd}, \mathrm{J}=32.5,13.6 \mathrm{~Hz}$, 2H), $3.61(\mathrm{dd}, \mathrm{J}=16.9,13.8 \mathrm{~Hz}, 4 \mathrm{H}), 3.37(\mathrm{~s}, 6 \mathrm{H}), 2.15(\mathrm{dd}, \mathrm{J}=13.8,6.8 \mathrm{~Hz}, 2 \mathrm{H}), 1.84-$ $1.70(\mathrm{~m}, 4 \mathrm{H}), 1.58-1.48(\mathrm{~m}, 2 \mathrm{H}), 1.44(\mathrm{dt}, \mathrm{J}=14.5,7.3 \mathrm{~Hz}, 2 \mathrm{H}), 1.00$ (t, J = 7.3 Hz, 3H). ${ }^{13} \mathrm{C} \mathrm{NMR} \mathrm{(126} \mathrm{MHz,} \mathrm{CDCl}_{3}$ ): $\delta 136.61$ (s), 114.87 (s), 62.99 (d, J = 11.9 Hz), 50.22 (s), 32.04 (s), 24.34 (s), 23.78 (s), 21.10 (s), 18.73 (s), 12.91 (s).

\section{$N$-butyl- $N$, $N$-dimethylhex-5-en-1-aminium chloride}




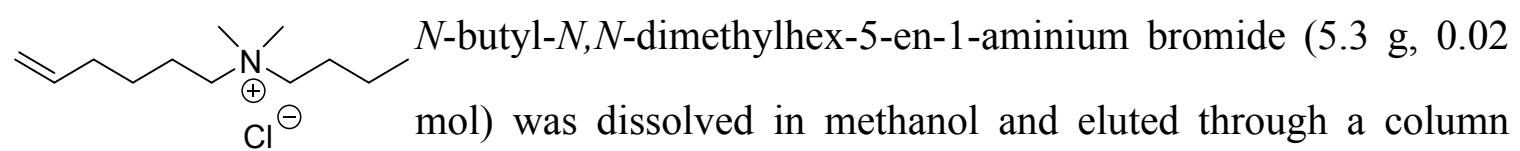

$\mathrm{Cl}^{\ominus}$ mol) was dissolved in methanol and eluted through a column containing Amberlite IRA-400 (Cl) ion-exchange resin (50.0 g)

at a drop rate of $1 \mathrm{~mL} / \mathrm{min}$. Solvent was removed under reduced pressure at $50{ }^{\circ} \mathrm{C}$, affording a tan solid $(4.0 \mathrm{~g}, 92 \%$ yield $) .{ }^{1} \mathrm{H} \mathrm{NMR}\left(500 \mathrm{MHz}, \mathrm{CDCl}_{3}\right): \delta 5.76(\mathrm{ddt}, \mathrm{J}=$ $16.9,10.2,6.7 \mathrm{~Hz}, 1 \mathrm{H}), 5.10-4.96(\mathrm{~m}, 2 \mathrm{H}), 3.61-3.51(\mathrm{~m}, 4 \mathrm{H}), 3.41(\mathrm{~s}, 6 \mathrm{H}), 2.19-$ $2.09(\mathrm{~m}, 2 \mathrm{H}), 1.78-1.63(\mathrm{~m}, 4 \mathrm{H}), 1.56-1.47(\mathrm{~m}, 2 \mathrm{H}), 1.47-1.37(\mathrm{~m}, 2 \mathrm{H}), 0.99(\mathrm{q}, \mathrm{J}=$ $7.0 \mathrm{~Hz}, 3 \mathrm{H}) .{ }^{13} \mathrm{C} \mathrm{NMR}\left(126 \mathrm{MHz}, \mathrm{CDCl}_{3}\right): \delta 137.38,115.97,63.63,63.56,51.23,33.02$, $25.34,24.75,22.03,19.75,13.80$.

\section{$N, N, N$-tripropylpent-4-en-1-aminium chloride}

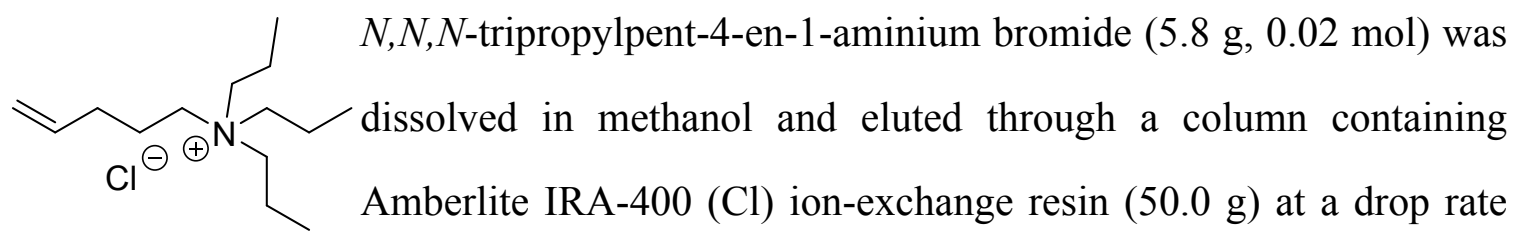
of $1 \mathrm{~mL} / \mathrm{min}$. Solvent was removed under reduced pressure at $50^{\circ} \mathrm{C}$, affording a white solid (4.8 g, 96\% yield). ${ }^{1} \mathrm{H} \mathrm{NMR}\left(500 \mathrm{MHz}, \mathrm{CDCl}_{3}\right): \delta 5.79$ (ddt, $\mathrm{J}=$ 17.0, 10.2, $6.8 \mathrm{~Hz}, 1 \mathrm{H}), 5.20-5.00(\mathrm{~m}, 2 \mathrm{H}), 3.47-3.33(\mathrm{~m}, 8 \mathrm{H}), 2.22(\mathrm{q}, \mathrm{J}=6.9 \mathrm{~Hz}$, 2H), $1.91-1.82(\mathrm{~m}, 2 \mathrm{H}), 1.82-1.71(\mathrm{~m}, 6 \mathrm{H}), 1.10-1.02(\mathrm{~m}, 9 \mathrm{H}) .{ }^{13} \mathrm{C}$ NMR $(126 \mathrm{MHz}$ $\left.\mathrm{CDCl}_{3}\right): \delta 135.76,117.16,60.60,58.51,30.08,21.33,15.78,10.85$.

\section{$N, N, N$-tributylpent-4-en-1-aminium chloride}

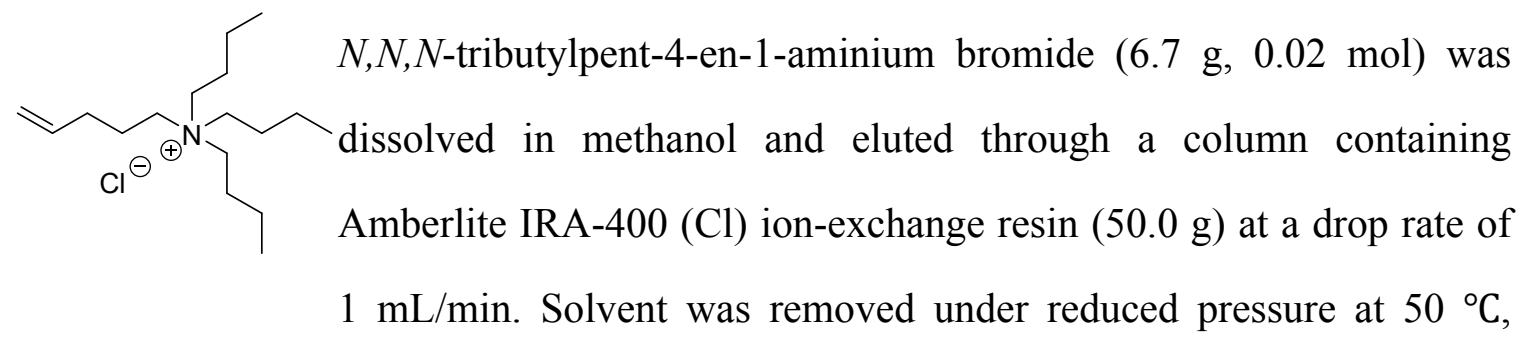

affording a tan solid (5.6 g, 96\% yield). ${ }^{1} \mathrm{H}$ NMR $\left(500 \mathrm{MHz}, \mathrm{CDCl}_{3}\right): \delta 5.79$ (ddt, $\mathrm{J}=$ 17.0, 10.2, $6.8 \mathrm{~Hz}, 1 \mathrm{H}), 5.19-5.06(\mathrm{~m}, 2 \mathrm{H}), 3.49-3.32(\mathrm{~m}, 8 \mathrm{H}), 2.23(\mathrm{dd}, \mathrm{J}=13.7,6.8$ 
$\mathrm{Hz}, 2 \mathrm{H}), 1.92-1.82(\mathrm{~m}, 2 \mathrm{H}), 1.74-1.62(\mathrm{~m}, 6 \mathrm{H}), 1.52-1.42(\mathrm{~m}, 6 \mathrm{H}), 1.06-0.97(\mathrm{~m}$, 9H). ${ }^{13} \mathrm{C}$ NMR $\left(126 \mathrm{MHz}, \mathrm{CDCl}_{3}\right): \delta 136.07,117.70,59.36,58.93,30.48,24.49,21.74$, $20.08,14.01$.

\section{Synthesis of organoboron complexes}

The organoboron complexes were obtained according to similar procedures in published works. ${ }^{1-3}$ To a solution of quaternary ammonium salt in THF was added the appropriate amount of a solution of 9-borabicyclo[3.3.1]nonane (9-BBN) in THF (0.5 M) via a cannula at room temperature in glovebox. The battle was sealed and heated to $60{ }^{\circ} \mathrm{C}$ for $12 \mathrm{~h}$ before cooling to room temperature. Then the mixture was concentrated in vacuo and purified by washing with $n$-hexane for three times. Wherein, white solid product was produced and next dried in vacuum at $30{ }^{\circ} \mathrm{C}$ to a constant weight (for $\mathbf{1 a} \sim \mathbf{1 c}$ ).

As for the rest complexes charged with benzoate $\left(\mathrm{PhCOO}^{-}\right)$or carboxylate $\left(\mathrm{CH}_{3} \mathrm{COO}^{-}\right)$, an extra step of anion replacement is needed. In glovebox, to a stirred solution of organobonron catalyst (charged with $\left.\mathrm{Cl}^{-}\right)$in $\mathrm{CH}_{2} \mathrm{Cl}_{2}(60 \mathrm{~mL})$ was added the appropriate amount of dried sodium benzoate (2.0 equiv.) or sodium acetate (2.0 equiv.). After the turbid mixture was stirred at room temperature for 2 days, it was filtered. Subsequently, the filtrate was concentrated and further purified by washing with n-hexane for purpose of getting rid of impurities, and dried under reduced pressure to furnish catalysts as white solids (for $\mathbf{1 d ~} \sim \mathbf{6}$ ).

\section{Catalyst 1d}

acetate $(0.8 \mathrm{~g}, 0.01 \mathrm{~mol}, 2.00$ equiv. $)$ were applied for anion
replacement. By subsequent filtration and purification, the 
catalyst was obtained as a white solid ( $1.5 \mathrm{~g}, 96 \%$ yield). ${ }^{1} \mathrm{H}$ NMR (400 MHz, $\left.\mathrm{CDCl}_{3}\right): \delta$ $3.44-3.37(\mathrm{~m}, 2 \mathrm{H}), 3.22-3.12(\mathrm{~m}, 2 \mathrm{H}), 3.02(\mathrm{~s}, 6 \mathrm{H}), 2.01(\mathrm{~s}, 3 \mathrm{H}), 1.93-1.65(\mathrm{~m}$, 10H), $1.58(\mathrm{~d}, \mathrm{~J}=7.1 \mathrm{~Hz}, 4 \mathrm{H}), 1.48-1.35(\mathrm{~m}, 4 \mathrm{H}), 1.02(\mathrm{t}, \mathrm{J}=7.4 \mathrm{~Hz}, 3 \mathrm{H}), 0.70(\mathrm{~s}, 2 \mathrm{H})$, $0.59(\mathrm{t}, \mathrm{J}=7.5 \mathrm{~Hz}, 2 \mathrm{H}) .{ }^{13} \mathrm{C} \mathrm{NMR}\left(101 \mathrm{MHz}, \mathrm{CDCl}_{3}\right): \delta 177.10,69.63,64.49,51.00$, $32.57,25.91,25.21,24.89,24.32,20.00,19.43,17.20,13.82$. HRMS (ESI): calcd for $\mathrm{C}_{17} \mathrm{H}_{35} \mathrm{BN}^{+}[\mathrm{M}-\mathrm{AcO}]^{+}$264.2857, found 264.2856.

\section{Catalyst 1e}

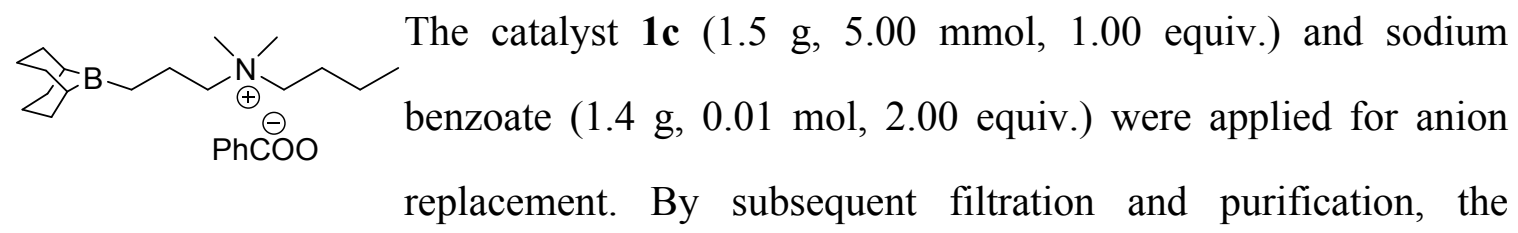

catalyst was obtained as a white solid $\left(1.9 \mathrm{~g}, 99 \%\right.$ yield). ${ }^{1} \mathrm{H} \mathrm{NMR}\left(400 \mathrm{MHz}, \mathrm{CDCl}_{3}\right): \delta$ $8.02(\mathrm{~d}, \mathrm{~J}=6.9 \mathrm{~Hz}, 2 \mathrm{H}), 7.30(\mathrm{dd}, \mathrm{J}=12.2,6.7 \mathrm{~Hz}, 3 \mathrm{H}), 3.36-3.28(\mathrm{~m}, 2 \mathrm{H}), 3.07-2.95$ (m, 2H), 2.87 (s, 6H), $1.81(\mathrm{~s}, 6 \mathrm{H}), 1.73-1.62(\mathrm{~m}, 2 \mathrm{H}), 1.52(\mathrm{t}, \mathrm{J}=10.0 \mathrm{~Hz}, 7 \mathrm{H}), 1.39$ (s, $3 \mathrm{H}), 0.84(\mathrm{t}, \mathrm{J}=7.3 \mathrm{~Hz}, 3 \mathrm{H}), 0.75(\mathrm{~s}, 2 \mathrm{H}), 0.67$ (t, J = 7.3 Hz, 2H). ${ }^{13} \mathrm{C} \mathrm{NMR}(101 \mathrm{MHz}$, $\left.\mathrm{CDCl}_{3}\right): \delta 169.22,135.58,129.65,128.36,126.82,68.25,63.19,49.68,31.51,26.28$, $24.79,24.40,23.48,18.60,18.39,12.45$. HRMS (ESI): calcd for $\mathrm{C}_{17} \mathrm{H}_{35} \mathrm{BN}^{+}$ $[\mathrm{M}-\mathrm{PhCOO}]^{+}$264.2857, found 264.2858.

\section{Catalyst 2}

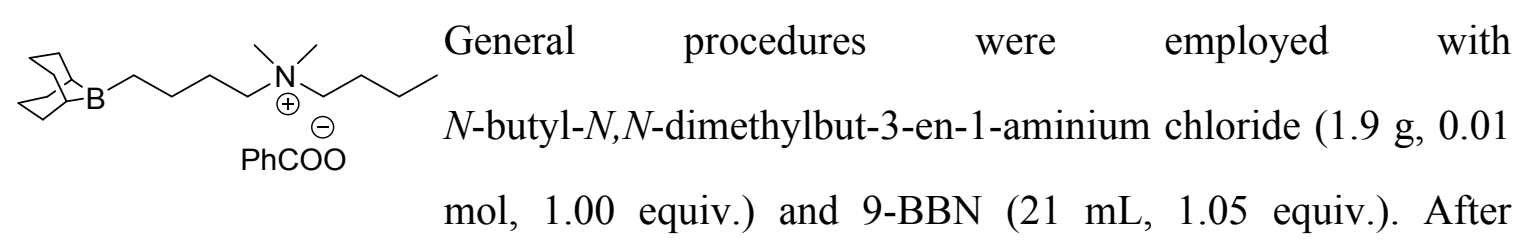

reaction and purification, the product was obtained as a white solid (3.1g, 100\% yield), and the product was used directly for the next step without further purification or characterizations. The white product $(1.6 \mathrm{~g}, 5.00 \mathrm{mmol}, 1.00$ equiv. $)$ and sodium 
benzoate (1.4 g, $0.01 \mathrm{~mol}, 2.00$ equiv.) were applied for anion replacement. By subsequent filtration and purification, the catalyst was obtained as a white solid (1.9 g, 97\% yield). ${ }^{1} \mathrm{H}$ NMR (400 MHz, $\left.\mathrm{CDCl}_{3}\right): \delta 8.03(\mathrm{~d}, \mathrm{~J}=6.7 \mathrm{~Hz}, 2 \mathrm{H}), 7.38-7.22(\mathrm{~m}, 3 \mathrm{H})$, $3.40-3.30(\mathrm{~m}, 2 \mathrm{H}), 3.07-2.93(\mathrm{~m}, 2 \mathrm{H}), 2.87$ (s, 6H), $1.81(\mathrm{~s}, 5 \mathrm{H}), 1.75-1.63(\mathrm{~m}, 3 \mathrm{H})$, $1.63-1.45(\mathrm{~m}, 7 \mathrm{H}), 1.39(\mathrm{~s}, 3 \mathrm{H}), 0.84(\mathrm{dd}, \mathrm{J}=15.1,7.7 \mathrm{~Hz}, 4 \mathrm{H}), 0.75(\mathrm{~s}, 3 \mathrm{H}), 0.68(\mathrm{t}, \mathrm{J}$ $=7.3 \mathrm{~Hz}, 2 \mathrm{H}) .{ }^{13} \mathrm{C} \mathrm{NMR}\left(101 \mathrm{MHz}, \mathrm{CDCl}_{3}\right): \delta 170.61,137.57,130.57,129.48,127.96$, $65.99,64.34,50.91,32.78,26.53,26.07,25.95,25.69,24.64,22.82,19.77,13.67$. HRMS (ESI): calcd for $\mathrm{C}_{18} \mathrm{H}_{37} \mathrm{BN}^{+}$[M-PhCOO] $]^{+}$278.3014, found 278.3015.

\section{Catalyst 3}

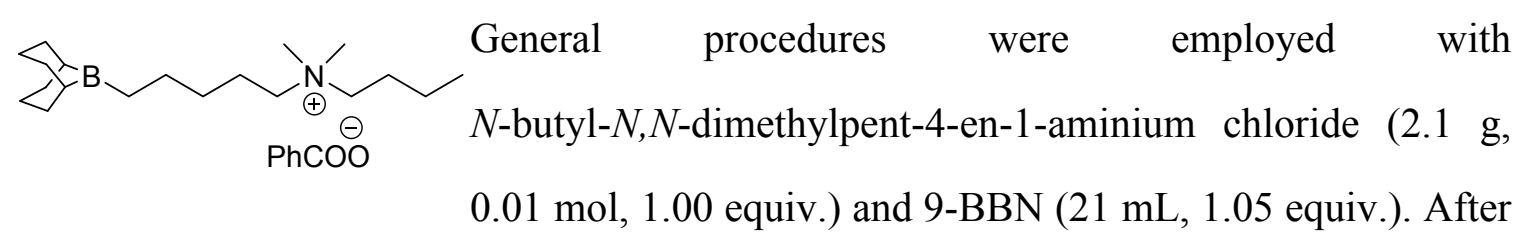
reaction and purification, the product was obtained as a white solid (3.2 $\mathrm{g}, 99 \%$ yield), and the product was used directly for the next step without further purification or characterizations. The white product $(1.6 \mathrm{~g}, 5.00 \mathrm{mmol}, 1.00$ equiv. $)$ and sodium benzoate (1.4 g, $0.01 \mathrm{~mol}, 2.00$ equiv.) were applied for anion replacement. By subsequent filtration and purification, the catalyst was obtained as a white solid ( $2.0 \mathrm{~g}$, 95\% yield). ${ }^{1} \mathrm{H}$ NMR (400 MHz, $\left.\mathrm{CDCl}_{3}\right): \delta 8.08-7.93(\mathrm{~m}, 2 \mathrm{H}), 7.39-7.22(\mathrm{~m}, 3 \mathrm{H})$, $3.23-3.14(\mathrm{~m}, 2 \mathrm{H}), 3.03(\mathrm{~s}, 2 \mathrm{H}), 3.00(\mathrm{~s}, 6 \mathrm{H}), 1.84(\mathrm{~s}, 5 \mathrm{H}), 1.80-1.66(\mathrm{~m}, 3 \mathrm{H}), 1.53(\mathrm{~d}$, $\mathrm{J}=4.7 \mathrm{~Hz}, 6 \mathrm{H}), 1.47-1.35(\mathrm{~m}, 4 \mathrm{H}), 1.30-1.23(\mathrm{~m}, 4 \mathrm{H}), 0.89(\mathrm{t}, \mathrm{J}=7.3 \mathrm{~Hz}, 3 \mathrm{H}), 0.77$ (s, 2H), $0.61-0.52(\mathrm{~m}, 2 \mathrm{H}) .{ }^{13} \mathrm{C}$ NMR $\left(101 \mathrm{MHz}, \mathrm{CDCl}_{3}\right): \delta$ 170.82, 137.86, 130.37, $129.63,127.86,59.61,58.72,52.99,33.34,32.93,29.65,27.60,26.00,24.22,23.96$, 21.02, 19.92, 13.73. HRMS (ESI): calcd for $\mathrm{C}_{19} \mathrm{H}_{39} \mathrm{BN}^{+}[\mathrm{M}-\mathrm{PhCOO}]^{+}$292.3170, found 292.3171. 


\section{Catalyst 4}

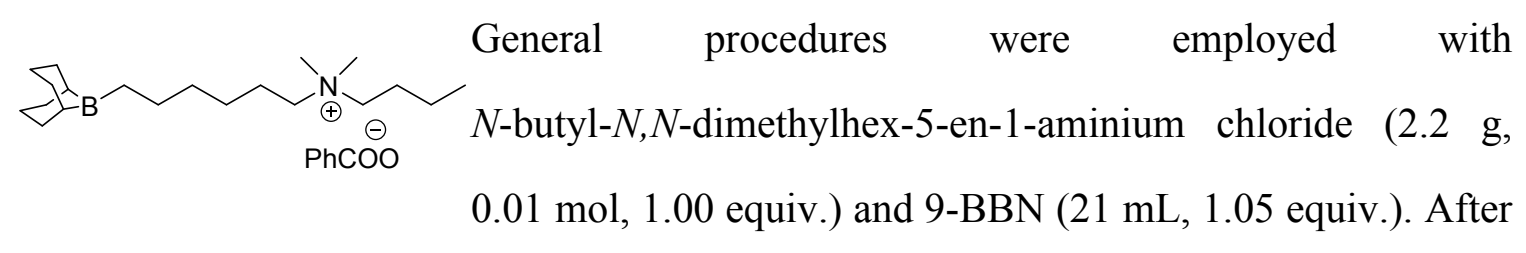

reaction and purification, the product was obtained as a white solid (3.3 g, 97\% yield), and the product was used directly for the next step without further purification or characterizations. The white product $(1.7 \mathrm{~g}, 5.00 \mathrm{mmol}, 1.00$ equiv. $)$ and sodium benzoate (1.4 g, $0.01 \mathrm{~mol}, 2.00$ equiv.) were applied for anion replacement. By subsequent filtration and purification, the catalyst was obtained as a white solid $(2.0 \mathrm{~g}$, 93\% yield). ${ }^{1} \mathrm{H}$ NMR (400 MHz, $\left.\mathrm{CDCl}_{3}\right): \delta 8.11-8.02(\mathrm{~m}, 2 \mathrm{H}), 7.35(\mathrm{~d}, \mathrm{~J}=7.3 \mathrm{~Hz}, 3 \mathrm{H})$, $3.33-3.27(\mathrm{~m}, 2 \mathrm{H}), 3.17-3.09(\mathrm{~m}, 2 \mathrm{H}), 3.03(\mathrm{~s}, 6 \mathrm{H}), 1.89(\mathrm{~s}, 6 \mathrm{H}), 1.60(\mathrm{~d}, \mathrm{~J}=7.2 \mathrm{~Hz}$, 8H), $1.43(\mathrm{~s}, 7 \mathrm{H}), 1.32(\mathrm{dd}, \mathrm{J}=14.9,7.4 \mathrm{~Hz}, 5 \mathrm{H}), 0.95(\mathrm{t}, \mathrm{J}=7.3 \mathrm{~Hz}, 3 \mathrm{H}), 0.72-0.64$ (m, 2H). $\left.{ }^{13} \mathrm{C} \mathrm{NMR} \mathrm{(101} \mathrm{MHz,} \mathrm{CDCl}_{3}\right): \delta 171.53,138.22,130.24,129.43,127.88,64.74$, $64.22,50.97,33.02,32.39,27.69,26.65,25.81,25.11,24.68,24.49,22.16,19.75,13.70$. HRMS (ESI): calcd for $\mathrm{C}_{20} \mathrm{H}_{41} \mathrm{BN}^{+}$[M-PhCOO] $^{+}$306.3327, found 306.3329.

\section{Catalyst 5}

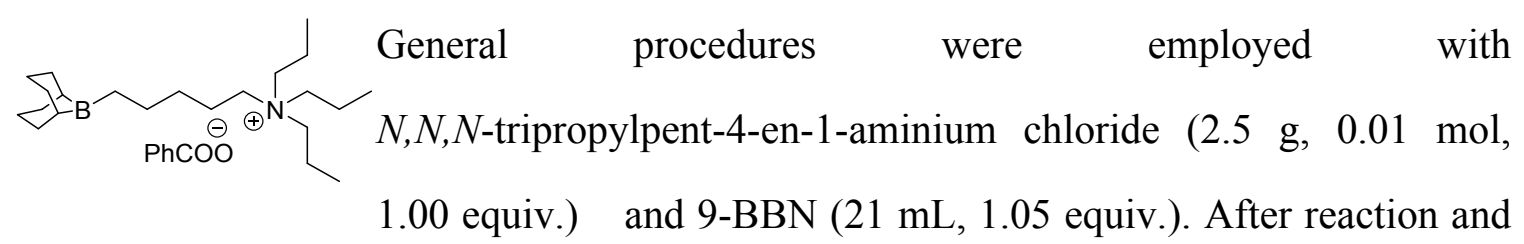
purification, the product was obtained as a white solid (3.6 g, 98\% yield), and the product was used directly for the next step without further purification or characterizations. The white product (1.8 g, $5.00 \mathrm{mmol}, 1.00$ equiv.) and sodium benzoate (1.4 g, $0.01 \mathrm{~mol}, 2.00$ equiv.) were applied for anion replacement. By subsequent filtration and purification, the catalyst was obtained as a white solid $(2.2 \mathrm{~g}, 96 \%$ yield $) .{ }^{1} \mathrm{H}$ NMR $\left(400 \mathrm{MHz}, \mathrm{CDCl}_{3}\right): \delta$ $8.11(\mathrm{dd}, \mathrm{J}=8.0,1.6 \mathrm{~Hz}, 2 \mathrm{H}), 7.35(\mathrm{dd}, \mathrm{J}=10.5,7.0 \mathrm{~Hz}, 3 \mathrm{H}), 3.23(\mathrm{~d}, \mathrm{~J}=17.2 \mathrm{~Hz}, 2 \mathrm{H})$, 
$3.07-2.97(\mathrm{~m}, 6 \mathrm{H}), 1.92(\mathrm{~s}, 6 \mathrm{H}), 1.66(\mathrm{ddd}, \mathrm{J}=26.5,16.9,8.4 \mathrm{~Hz}, 16 \mathrm{H}), 1.48(\mathrm{dd}, \mathrm{J}=$ 13.2, $6.7 \mathrm{~Hz}, 4 \mathrm{H}), 1.32(\mathrm{~d}, \mathrm{~J}=5.2 \mathrm{~Hz}, 2 \mathrm{H}), 1.00(\mathrm{t}, \mathrm{J}=7.3 \mathrm{~Hz}, 9 \mathrm{H}) .{ }^{13} \mathrm{C} \mathrm{NMR}(101 \mathrm{MHz}$, $\left.\mathrm{CDCl}_{3}\right): \delta 169.57,137.69,130.15,129.44,127.66,60.30,59.58,29.29,26.14,25.89$, 23.91, 20.55, 20.28, 15.48, 10.74. HRMS (ESI): calcd for $\mathrm{C}_{22} \mathrm{H}_{45} \mathrm{BN}^{+}[\mathrm{M}-\mathrm{PhCOO}]^{+}$ 334.3640, found 334.3640 .

\section{Catalyst 6}

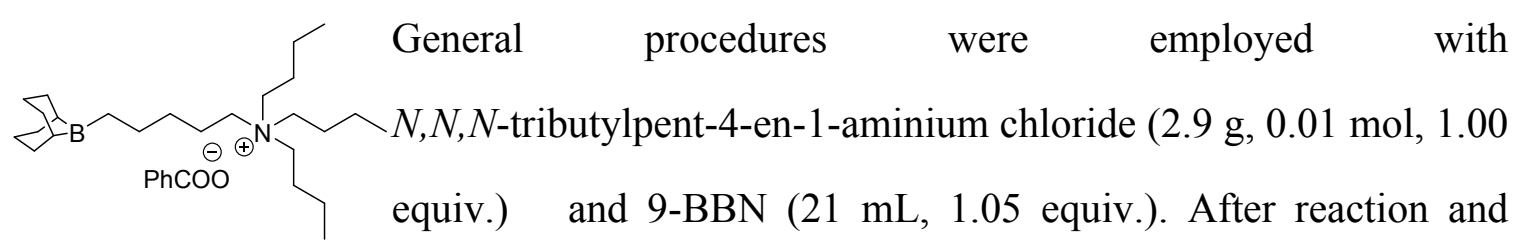
purification, the product was obtained as a white solid (4.1g, 99\%

yield), and the product was used directly for the next step without further purification or characterizations. The white product $(2.1 \mathrm{~g}, 5.00 \mathrm{mmol}, 1.00$ equiv. $)$ and sodium benzoate (1.4 g, $0.01 \mathrm{~mol}, 2.00$ equiv.) were applied for anion replacement. By subsequent to filtration and purification, the catalyst was obtained as a white solid ( $2.4 \mathrm{~g}$, 95\% yield). ${ }^{1} \mathrm{H}$ NMR (400 MHz, $\left.\mathrm{CDCl}_{3}\right): \delta 8.11(\mathrm{~d}, \mathrm{~J}=6.7 \mathrm{~Hz}, 2 \mathrm{H}), 7.34(\mathrm{dd}, \mathrm{J}=11.5$, $7.1 \mathrm{~Hz}, 3 \mathrm{H}), 3.30-3.20$ (m, 2H), $3.15-2.99$ (m, 6H), 1.90 (d, J = 12.5 Hz, 6H), 1.63 (dd, J = 22.5, $6.5 \mathrm{~Hz}, 14 \mathrm{H}), 1.51-1.44(\mathrm{~m}, 4 \mathrm{H}), 1.38(\mathrm{dt}, \mathrm{J}=14.7,7.4 \mathrm{~Hz}, 8 \mathrm{H}), 1.00$ (t, J $=7.3 \mathrm{~Hz}, 9 \mathrm{H}), 0.64(\mathrm{~d}, \mathrm{~J}=8.6 \mathrm{~Hz}, 2 \mathrm{H}) .{ }^{13} \mathrm{C} \mathrm{NMR}\left(101 \mathrm{MHz}, \mathrm{CDCl}_{3}\right): \delta 170.72,137.88$, $130.38,129.64,127.87,59.62,58.73,32.96,29.67,26.02,24.26,23.97,21.04,19.93$, 13.74. HRMS (ESI): calcd for $\mathrm{C}_{25} \mathrm{H}_{51} \mathrm{BN}^{+}[\mathrm{M}-\mathrm{PhCOO}]^{+} 376.4109$, found 376.4108 .

\section{NMR spectra of bifunctional organoboron catalysts}



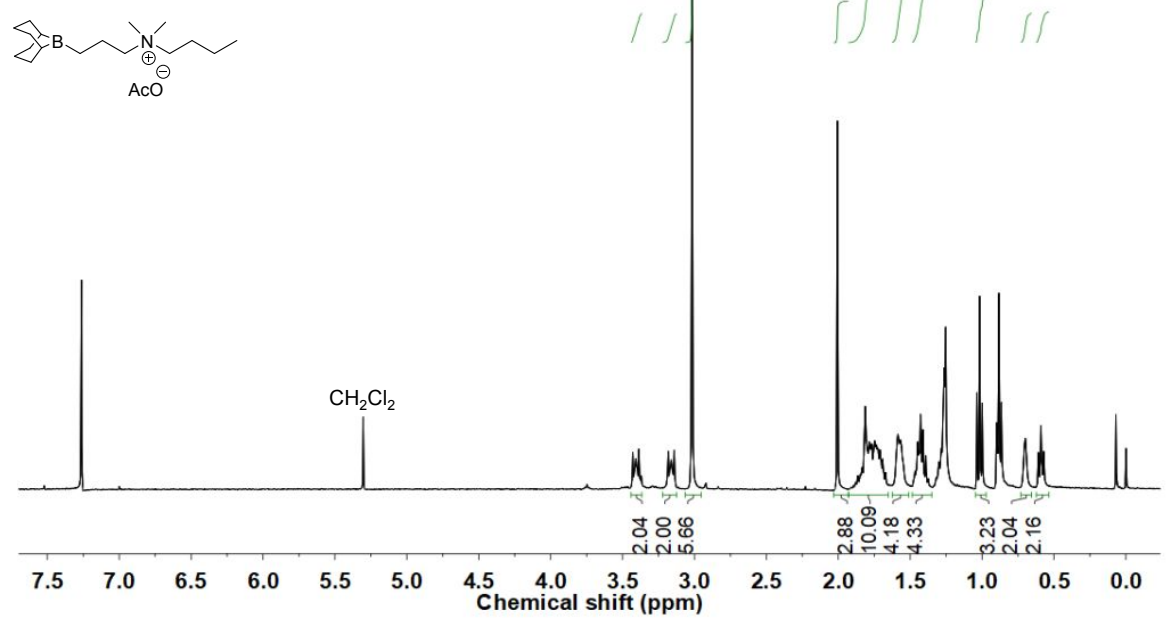

Figure S1. ${ }^{1} \mathrm{H}$ NMR spectrum of catalyst $1 d$ in $\mathrm{CDCl}_{3}$.

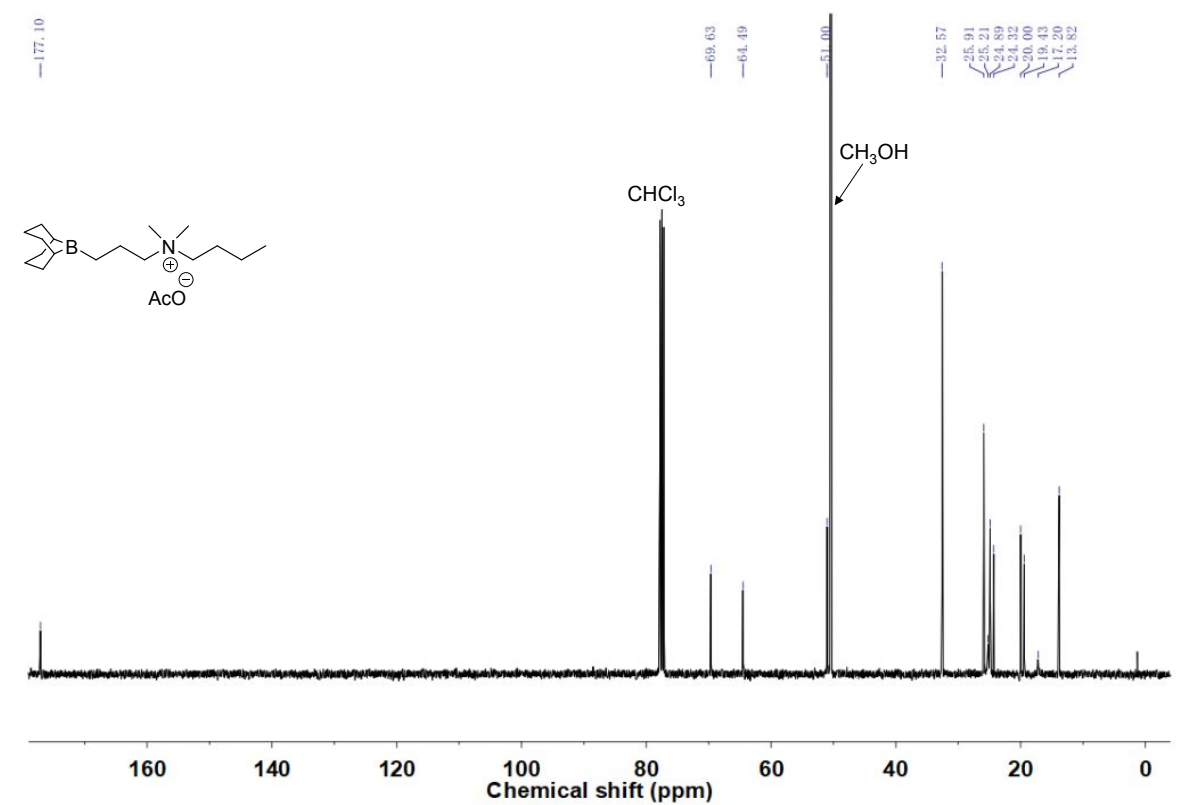

Figure S2. ${ }^{13} \mathrm{C}$ NMR spectrum of catalyst $1 \mathbf{d}$ in $\mathrm{CDCl}_{3}$. 


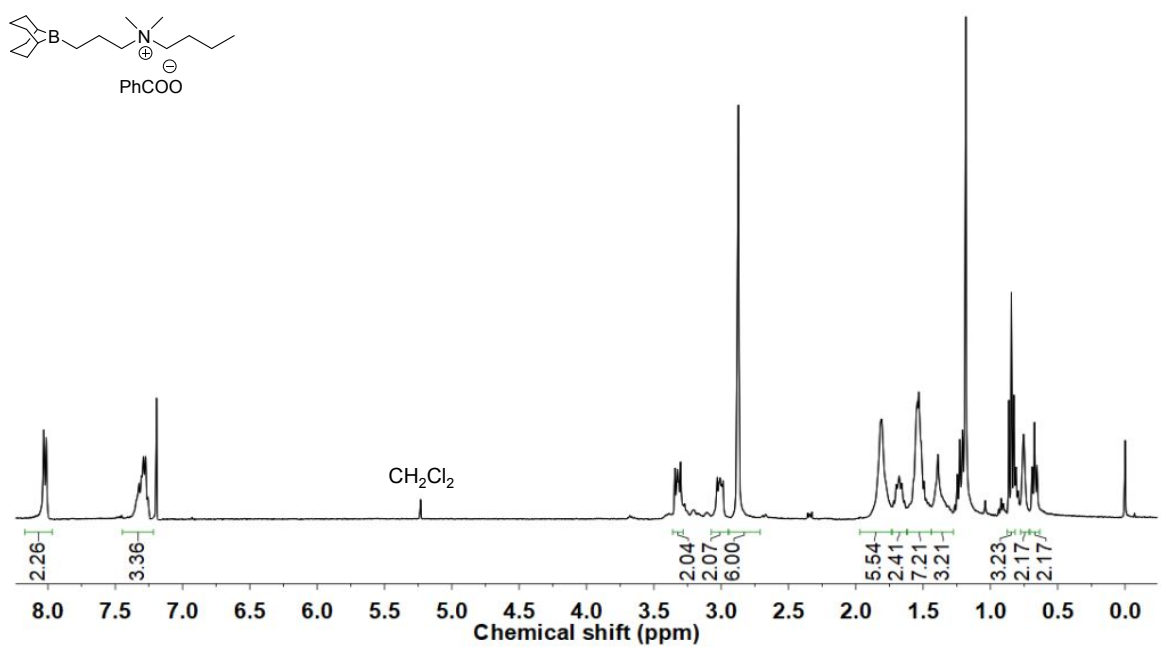

Figure S3. ${ }^{1} \mathrm{H}$ NMR spectrum of catalyst $1 \mathrm{e}$ in $\mathrm{CDCl}_{3}$.

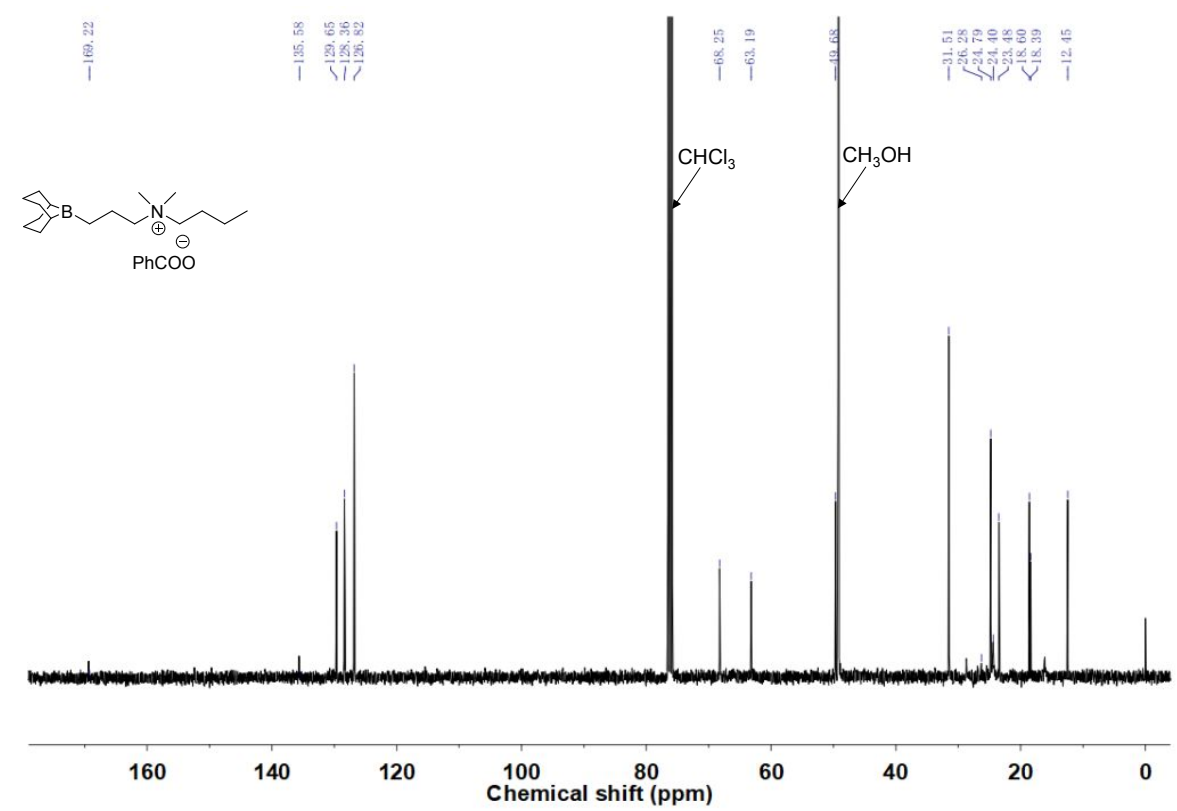

Figure S4. ${ }^{13} \mathrm{C}$ NMR spectrum of catalyst $1 \mathrm{e}$ in $\mathrm{CDCl}_{3}$. 

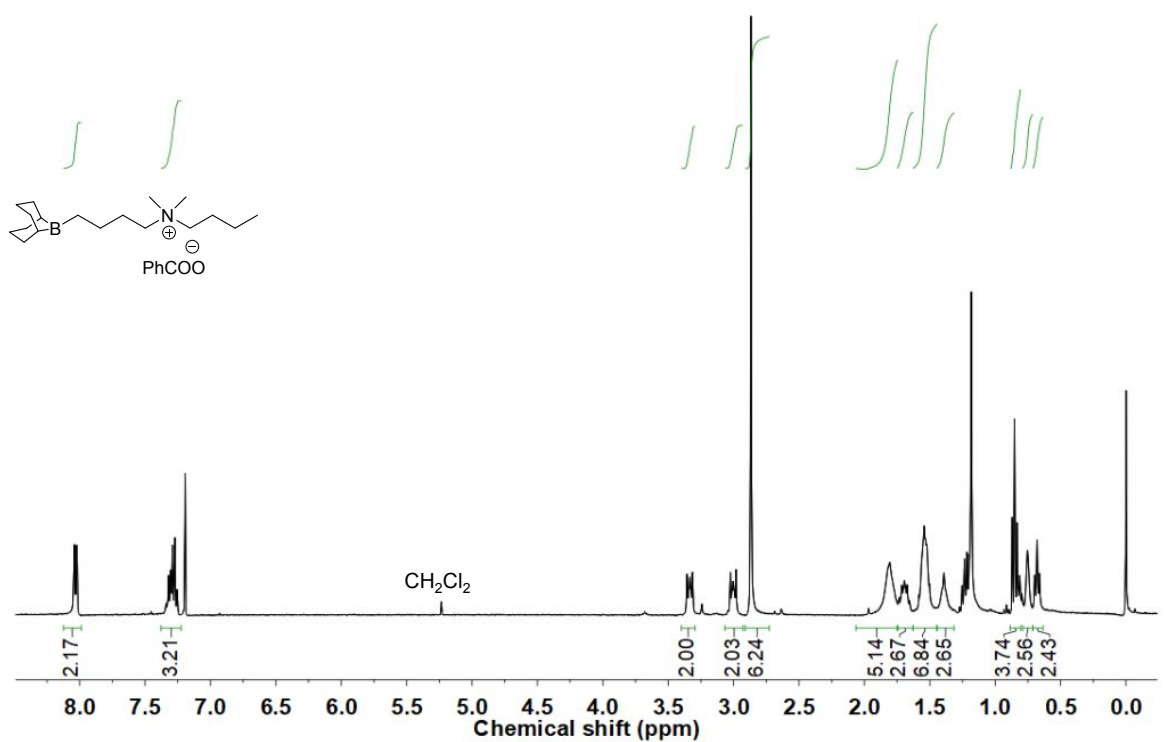

Figure S5. ${ }^{1} \mathrm{H}$ NMR spectrum of catalyst 2 in $\mathrm{CDCl}_{3}$.

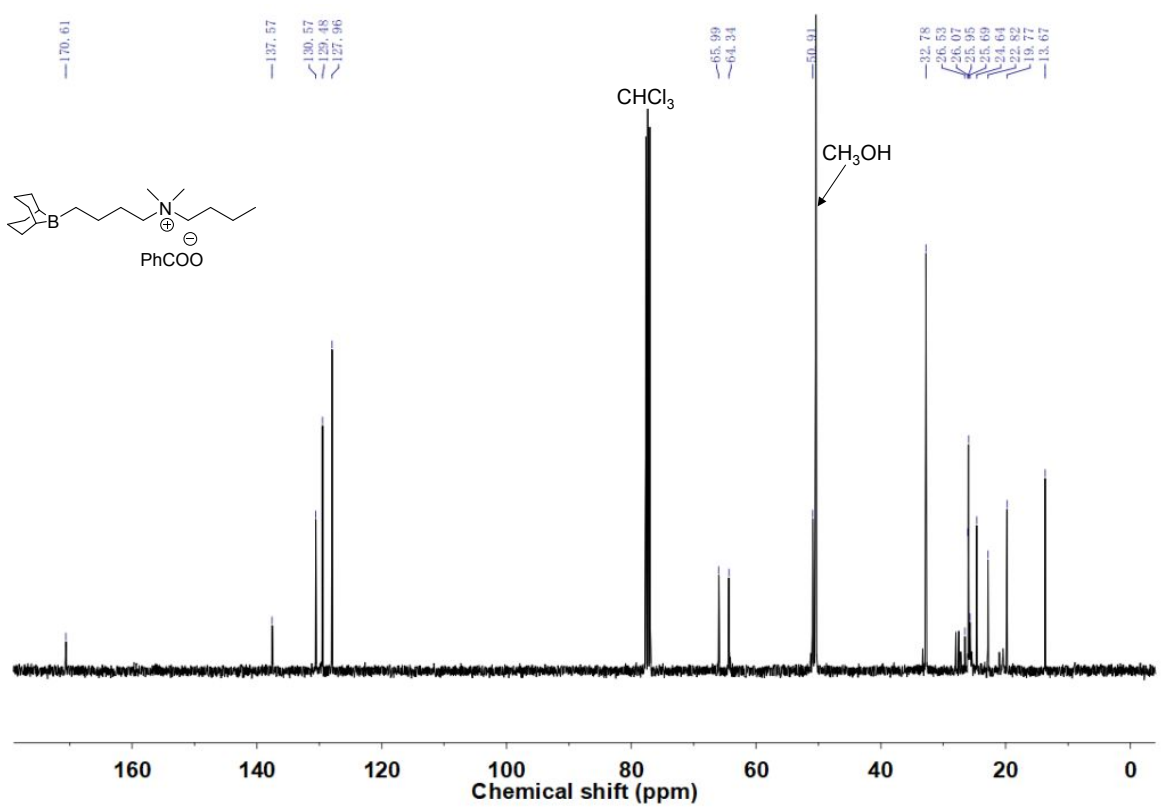

Figure S6. ${ }^{13} \mathrm{C}$ NMR spectrum of catalyst 2 in $\mathrm{CDCl}_{3}$. 

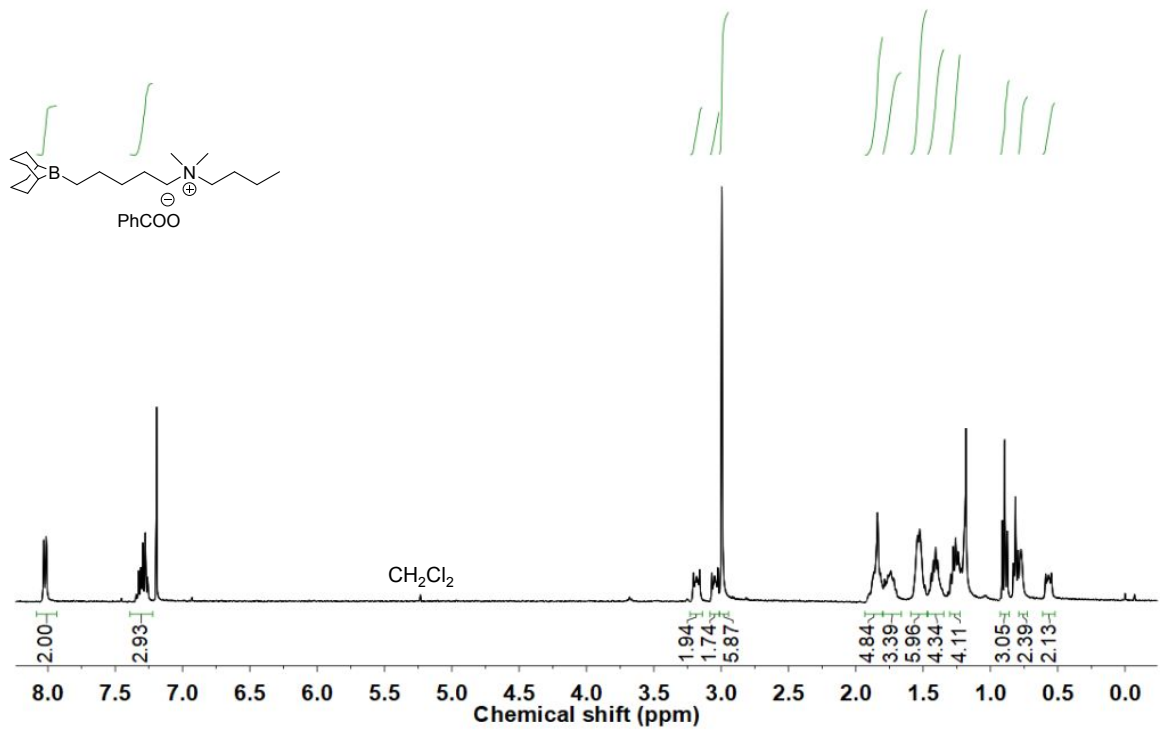

Figure S7. ${ }^{1} \mathrm{H}$ NMR spectrum of catalyst 3 in $\mathrm{CDCl}_{3}$.

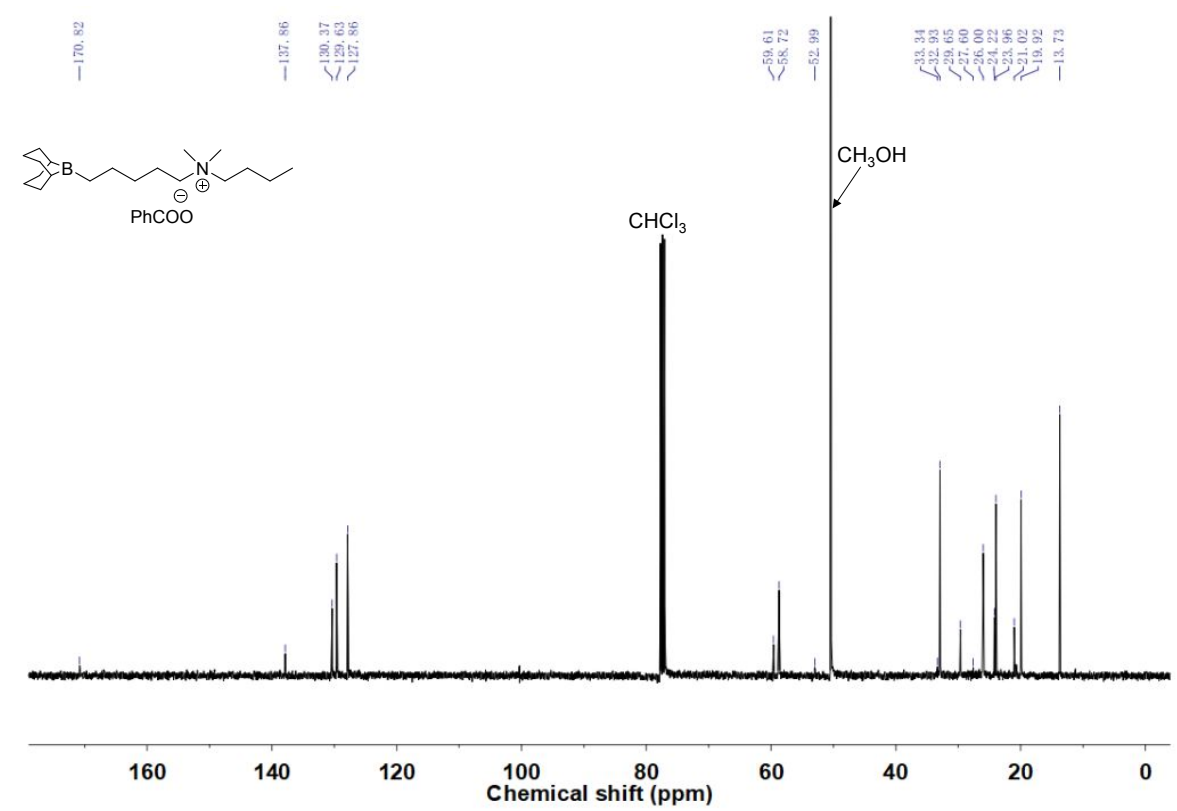

Figure S8. ${ }^{13} \mathrm{C}$ NMR spectrum of catalyst 3 in $\mathrm{CDCl}_{3}$. 

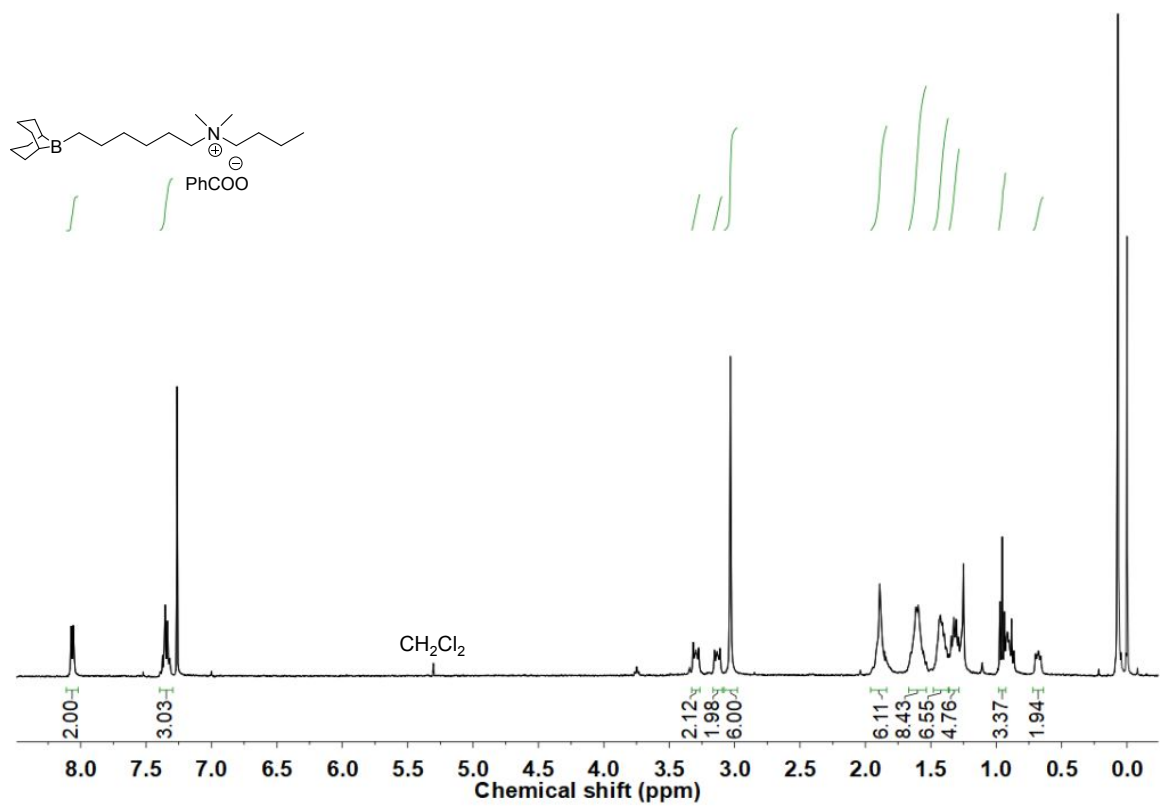

Figure S9. ${ }^{1} \mathrm{H}$ NMR spectrum of catalyst 4 in $\mathrm{CDCl}_{3}$.

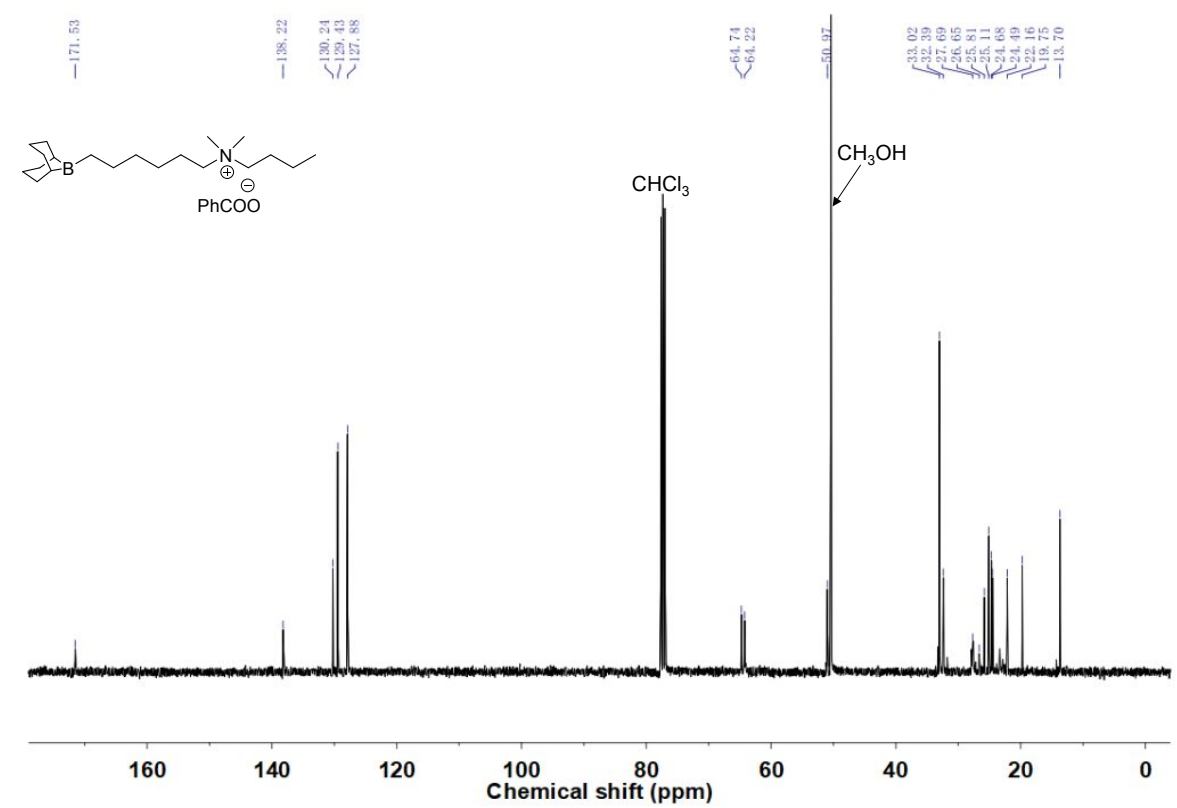

Figure S10. ${ }^{13} \mathrm{C}$ NMR spectrum of catalyst 4 in $\mathrm{CDCl}_{3}$. 


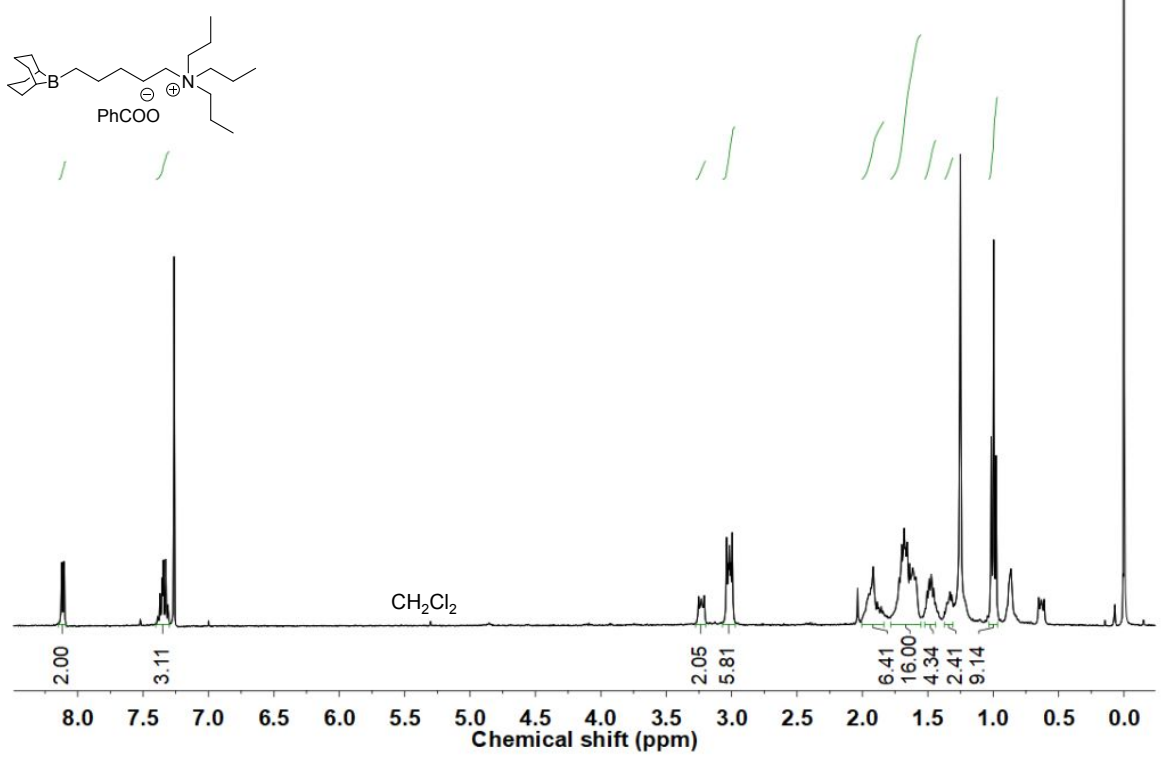

Figure S11. ${ }^{1} \mathrm{H}$ NMR spectrum of catalyst 5 in $\mathrm{CDCl}_{3}$.

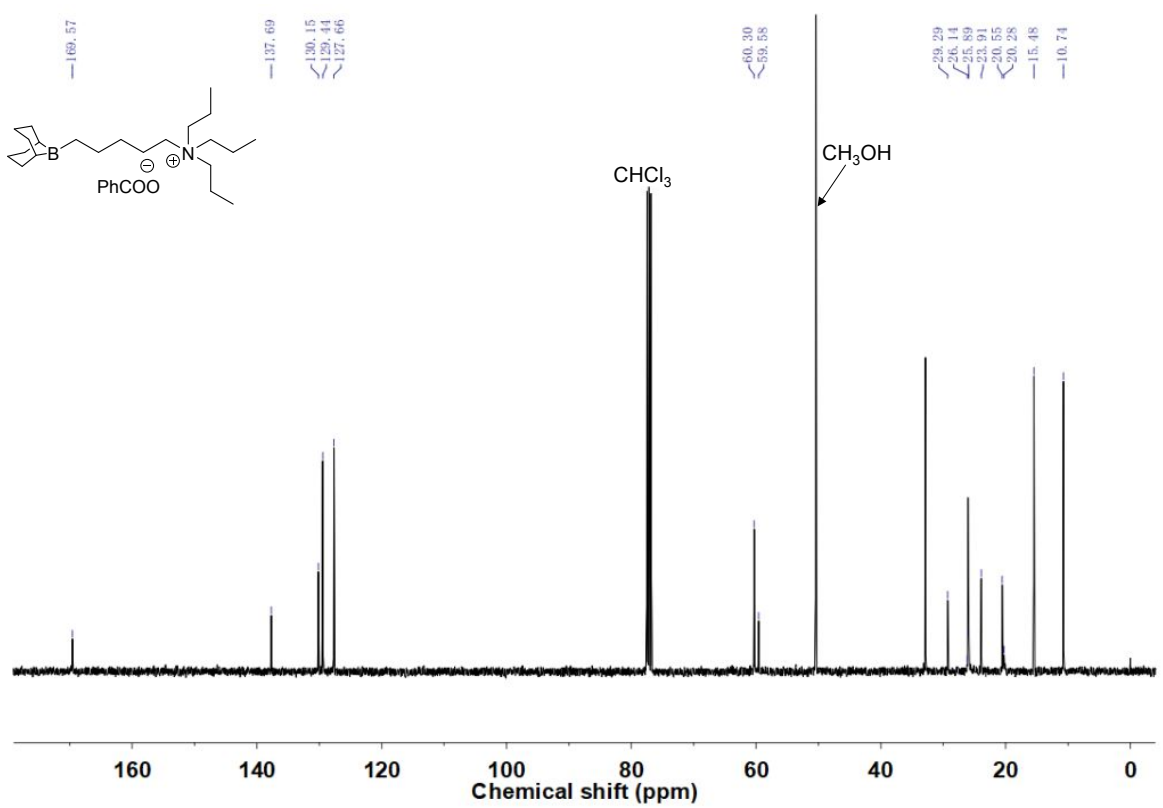

Figure S12. ${ }^{13} \mathrm{C}$ NMR spectrum of catalyst 5 in $\mathrm{CDCl}_{3}$. 

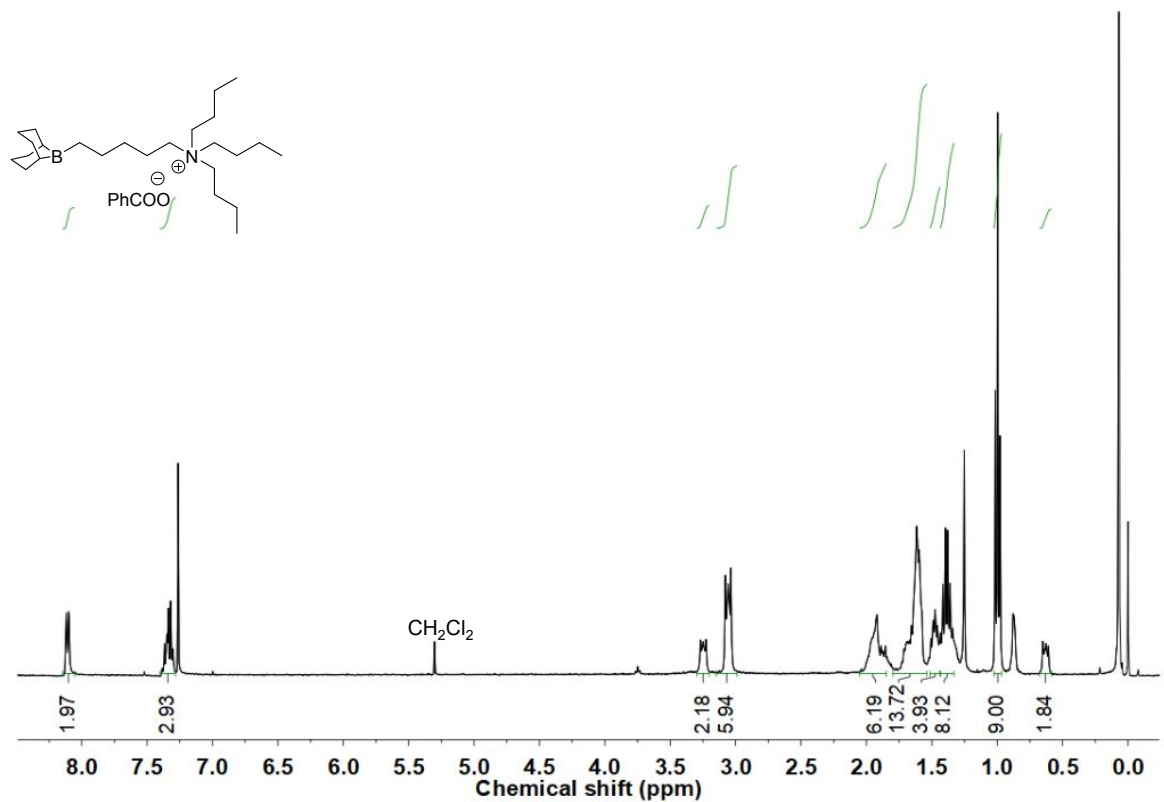

Figure S13. ${ }^{1} \mathrm{H}$ NMR spectrum of catalyst 6 in $\mathrm{CDCl}_{3}$.

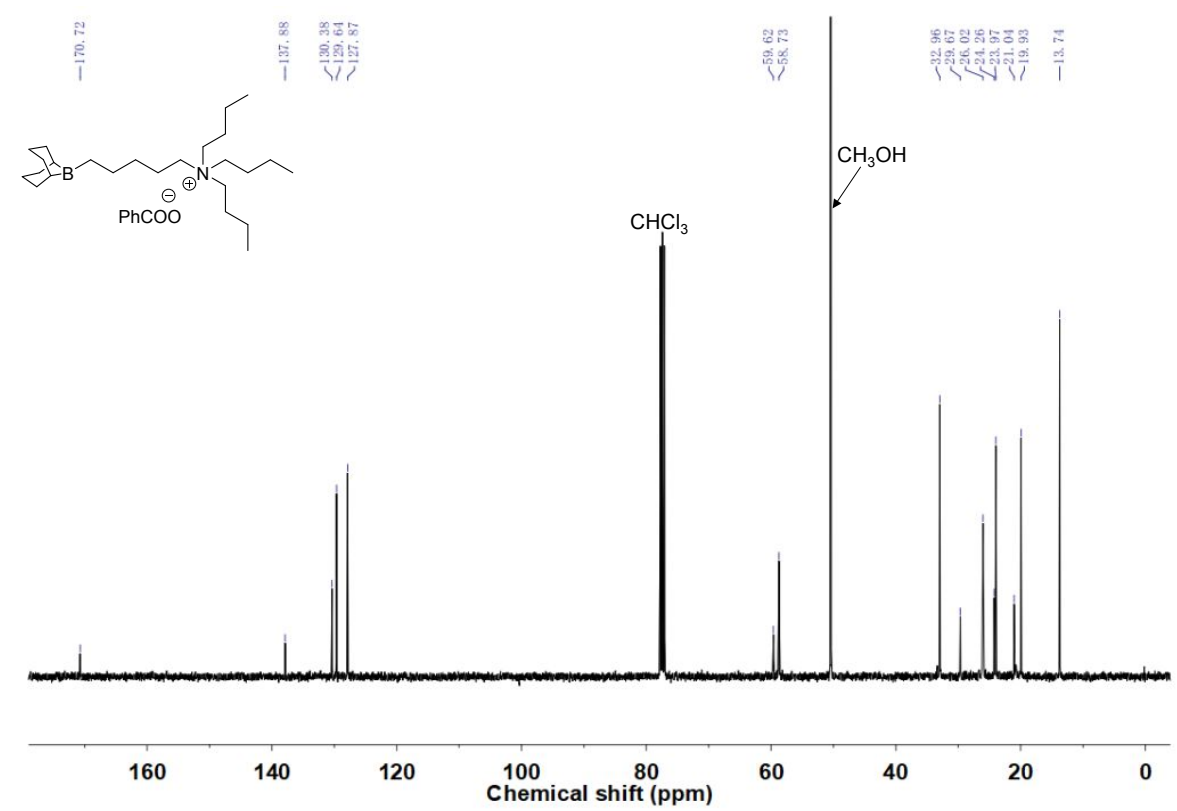

Figure S14. ${ }^{13} \mathrm{C}$ NMR spectrum of catalyst 6 in $\mathrm{CDCl}_{3}$. 


\section{Crystal data and structure refinement for $1 \mathrm{e}$}

Table S1 Crystal data and structure refinement for $1 \mathrm{e}$.

Empirical formula

Formula weight

Temperature/K

Crystal system

Space group

$\mathrm{a} / \AA$

$\mathrm{b} / \AA$

$\mathrm{c} / \AA$

$\alpha /{ }^{\circ}$

$\beta /{ }^{\circ}$

$\gamma / \circ$

Volume $/ \AA^{3}$

Z

$\rho_{\text {calc }} \mathrm{g} / \mathrm{cm}^{3}$

$\mu / \mathrm{mm}^{-1}$

$\mathrm{F}(000)$

Crystal size $/ \mathrm{mm}^{3}$

Radiation

$2 \Theta$ range for data collection $/{ }^{\circ} 4.73$ to 54.384

Index ranges

Reflections collected

Independent reflections

Data/restraints/parameters

Goodness-of-fit on $\mathrm{F}^{2}$

Final $\mathrm{R}$ indexes $[\mathrm{I}>=2 \sigma(\mathrm{I})]$

Final $\mathrm{R}$ indexes [all data]

Largest diff. peak/hole / e $\AA^{-3} 0.74 /-0.51$

$\mathrm{C}_{24} \mathrm{H}_{40} \mathrm{BNO}_{2}$

385.38

170.0

monoclinic

$\mathrm{P} 2{ }_{1} / \mathrm{n}$

$14.8723(11)$

9.8390(9)

$16.6022(12)$

90

$113.386(3)$

90

2229.8(3)

4

1.148

0.070

848.0

$0.29 \times 0.19 \times 0.06$

16436

$4925 / 4 / 275$

1.056

$\operatorname{MoK} \alpha(\lambda=0.71073)$

$-19 \leq \mathrm{h} \leq 18,-12 \leq \mathrm{k} \leq 11,-20 \leq 1 \leq 21$

$4925\left[\mathrm{R}_{\mathrm{int}}=0.0541, \mathrm{R}_{\text {sigma }}=0.0482\right]$

$\mathrm{R}_{1}=0.0640, \mathrm{wR}_{2}=0.1629$

$\mathrm{R}_{1}=0.0811, \mathrm{wR}_{2}=0.1773$ 


\section{General Polymerization Procedure}

In glovebox, a small vial was charged with a predetermined amount of catalyst and BBL. The reaction solution was perfectly mixed and next transferred to an oven-dried 10 $\mathrm{mL}$ Schlenk brittle sealed by a Teflon cap and equipped with a magnetic stir bar. The brittle was then quickly removed from the glovebox and immediately submerged into a temperature-controlled bath preheated to predetermined temperature. After the reaction mixture was stirred over the appropriate reaction time, the bottle was cooled below the freezing point and an aliquot was removed to determine the conversion via ${ }^{1} \mathrm{H}$ NMR spectrum, and the reaction was terminated by a few drops of methanol acidified with $\mathrm{HCl}$ ( $1 \mathrm{~mol} / \mathrm{L}$ ) and exposure to air. The quenched mixture was dissolved in $\mathrm{CH}_{2} \mathrm{Cl}_{2}$ and precipitated into $80 \mathrm{~mL}$ of petroleum ether, filtered, along with washed by petroleum ether. In the end, it was dried in a vacuum oven and the acquired products were then analyzed by NMR, GPC, and MALDI-TOF.

\section{Representative ${ }^{1} \mathrm{H}$ NMR spectra}

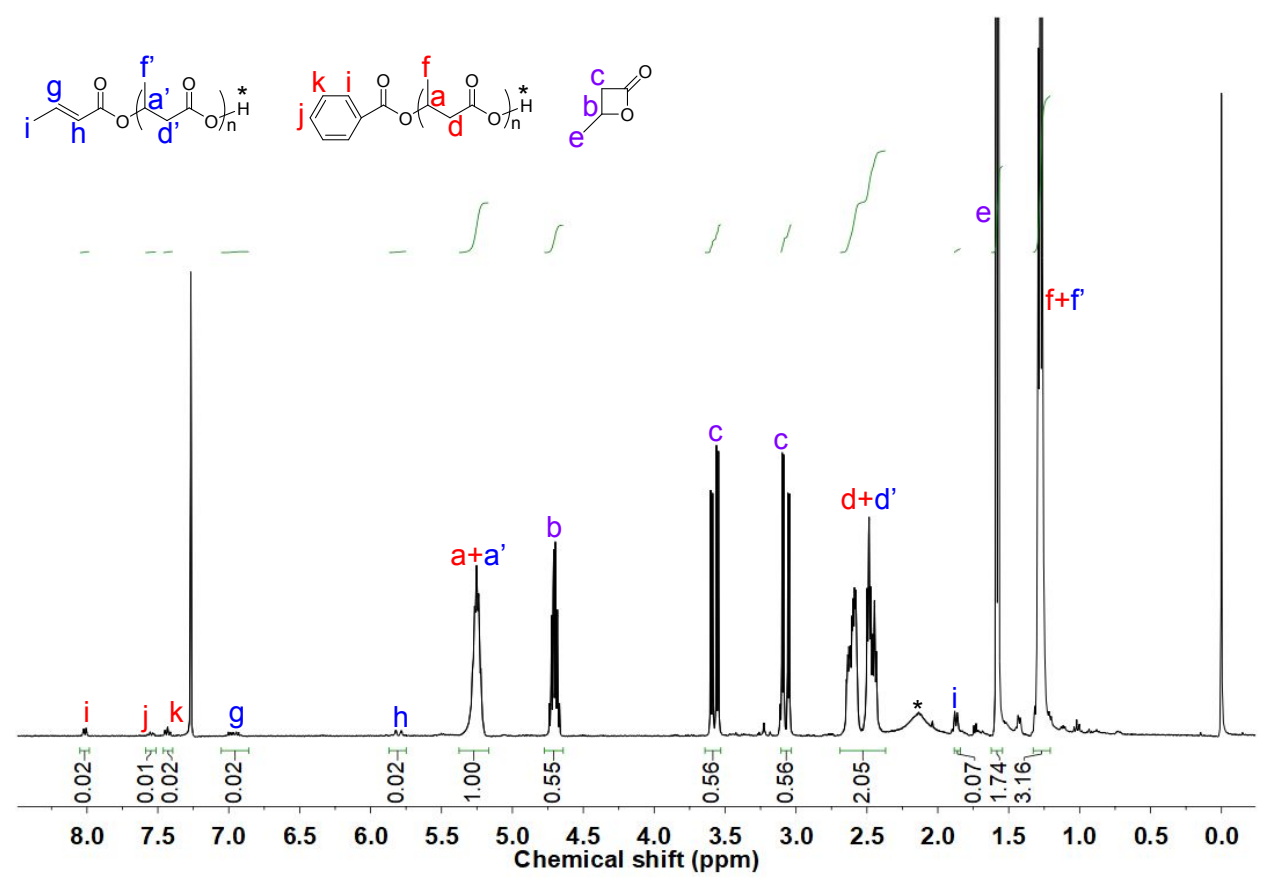

Figure S15. Representative ${ }^{1} \mathrm{H}$ NMR spectrum of a reaction mixture in $\mathrm{CDCl}_{3}\left(400 \mathrm{MHz}, 25^{\circ} \mathrm{C}\right)$. The polymerization was carried out at $100^{\circ} \mathrm{C}$ with $\mathrm{rac}$-BBL/3 $=200$ within $1 \mathrm{~h}$. (Table 1 , entry 15). 


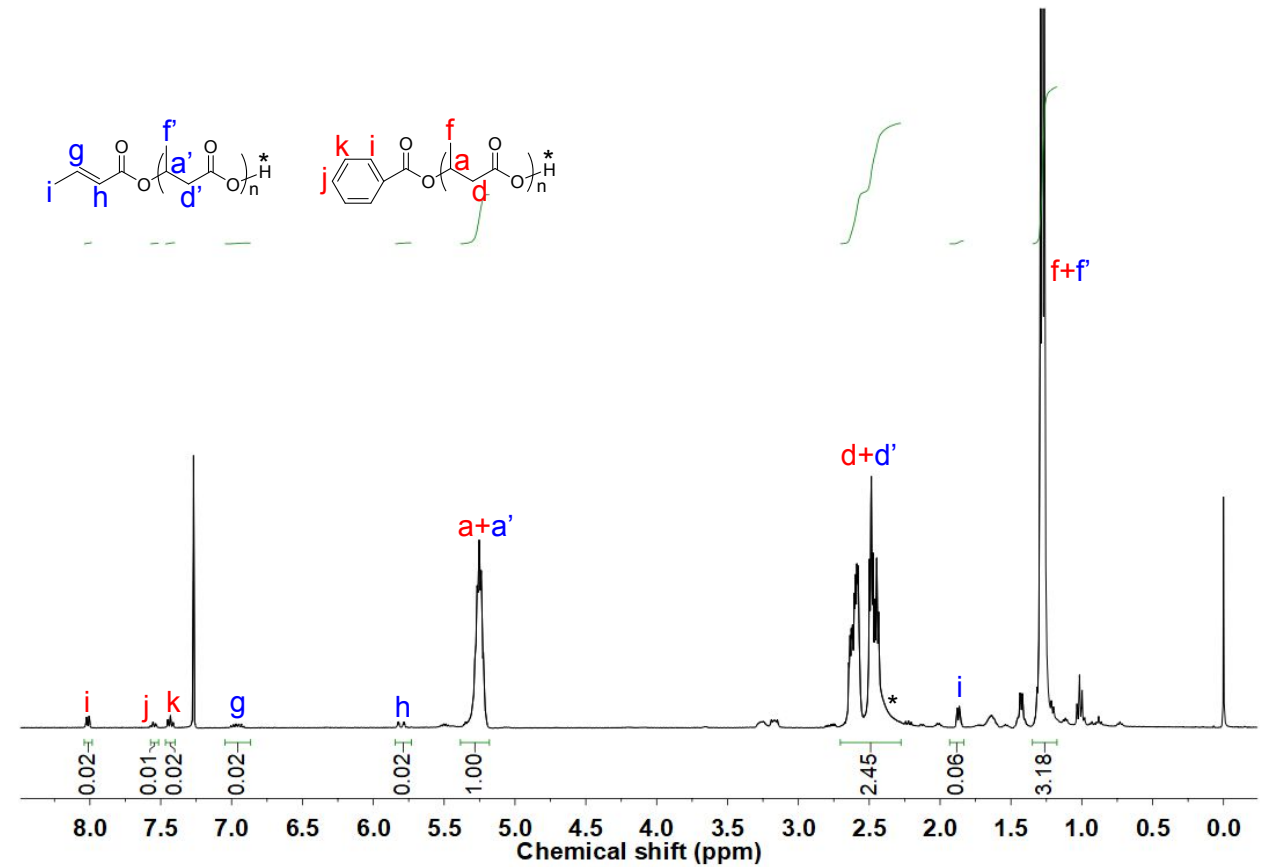

Figure S16. Representative ${ }^{1} \mathrm{H}$ NMR spectrum of a purified PHB sample in $\mathrm{CDCl}_{3}\left(400 \mathrm{MHz}, 25^{\circ} \mathrm{C}\right)$. The polymerization was carried out at $100{ }^{\circ} \mathrm{C}$ with $\mathrm{rac}-\mathrm{BBL} / \mathbf{6}=100$ within $1 \mathrm{~h}$ (Table 1 , entry 10).

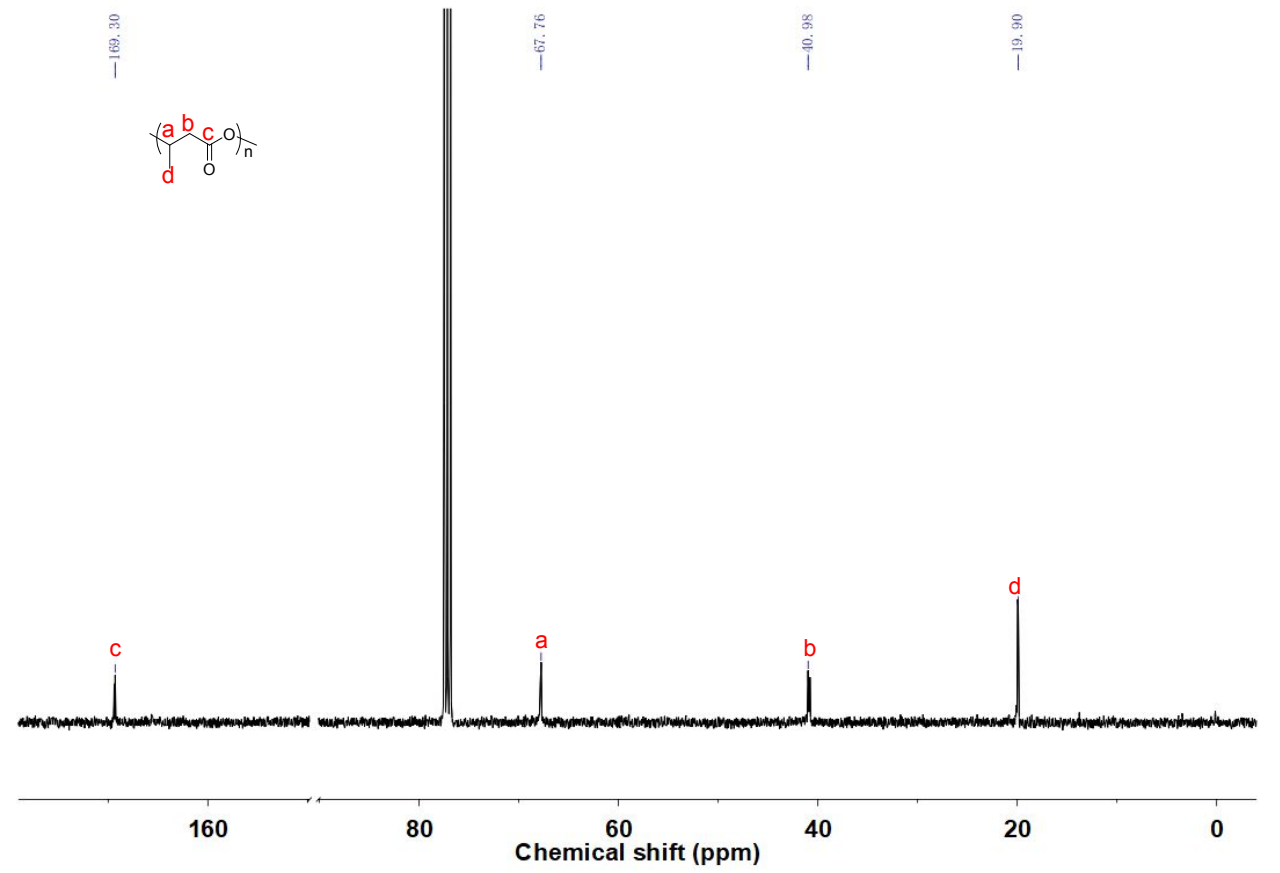

Figure S17. Representative ${ }^{13} \mathrm{C}$ NMR spectrum of the PHB sample in $\mathrm{CDCl}_{3}\left(101 \mathrm{MHz}, 25{ }^{\circ} \mathrm{C}\right)$. The polymerization was carried out at $100^{\circ} \mathrm{C}$ with $\mathrm{rac}-\mathrm{BBL} / \mathbf{6}=100$ within $1 \mathrm{~h}$ (Table 1 , entry 10). 


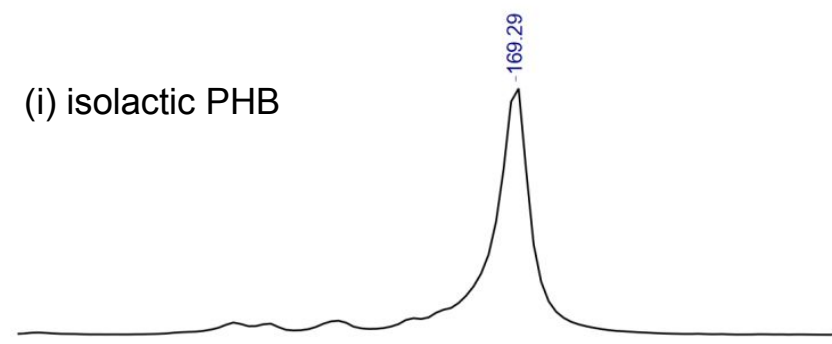

(ii) atactic $\mathrm{PHB}$

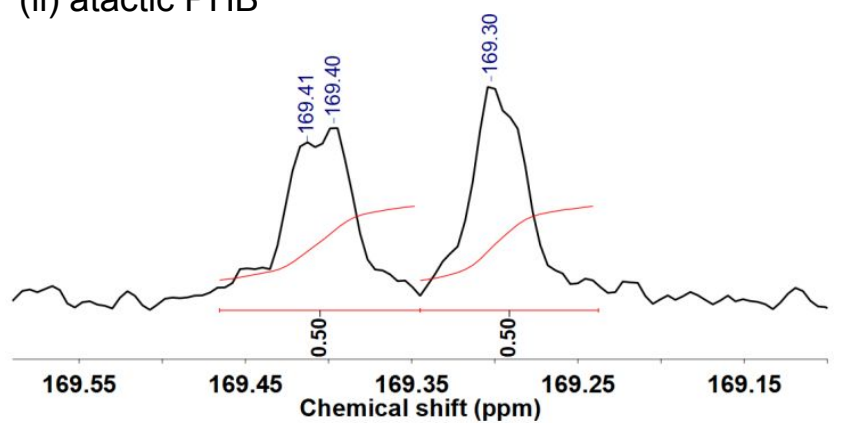

Figure S18. Carbonyl region of the ${ }^{13} \mathrm{C}$ NMR spectra of PHB prepared by the polymerization of (i) (S)-BBL and (ii) rac-BBL.

\section{Representative DSC curves of atactic and isotactic PHBs}
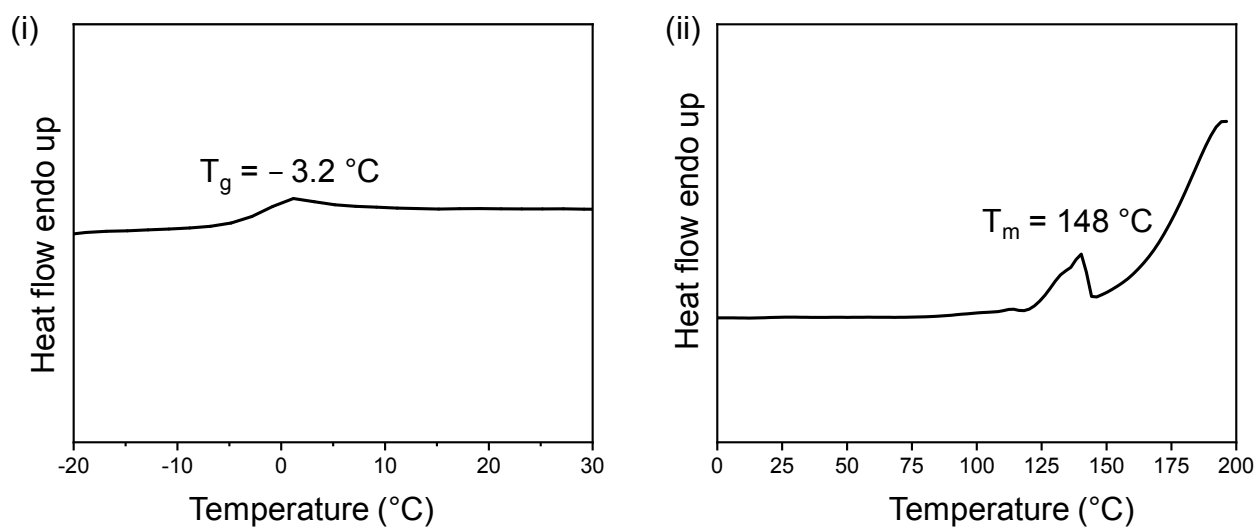

Figure S19. Representative DSC curves of (i) atactic PHB and (ii) isotactic PHB.

\section{The determination of carboxylate end-group by diphenylchlorophosphate ((PhO $\left.)_{2} \mathrm{POCl}\right)$.}

In glovebox, $3(0.023 \mathrm{mmol}, 9.6 \mathrm{mg})$ was introduced into a small vial before 
rac-BBL (1.16 mmol, $0.1 \mathrm{~g})$ was added. The reaction solution was perfectly mixed and next transferred to an oven-dried $10 \mathrm{~mL}$ Schlenk brittle sealed by a Teflon cap and equipped with a magnetic stir bar. The brittle was then quickly removed from the glovebox. The polymerization proceeded for $45 \mathrm{~min}$, and the bottle was cooled below the freezing point, then deuterated benzene $(0.55 \mathrm{~mL})$ was added followed by $(\mathrm{PhO})_{2} \mathrm{POCl}$ $(0.024 \mathrm{mmol}, 5 \mu \mathrm{L})$. After stirring for $1 \mathrm{~h}$, the mixture was transferred and analyzed by ${ }^{31} \mathrm{P}$ NMR spectroscopy.

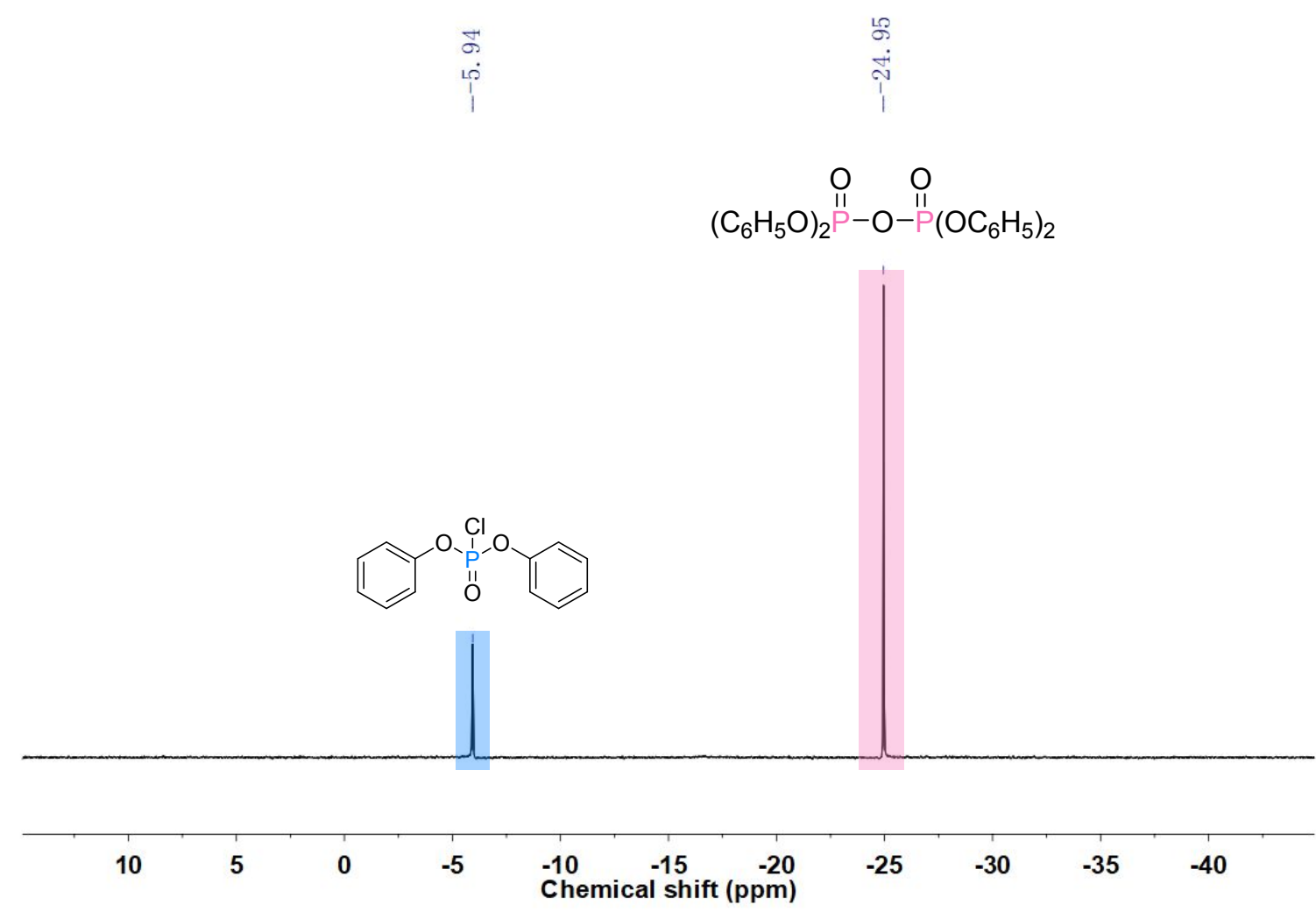

Figure S20. ${ }^{31} \mathrm{P}$ NMR spectrum in $\mathrm{C}_{6} \mathrm{D}_{6}$ obtained from the addition of excess of $(\mathrm{PhO})_{2} \mathrm{POCl}$ into the polymerization reaction mixture of $r a c$-BBL. Polymerization conditions: $r a c-B B L / 3=50,100{ }^{\circ} \mathrm{C}, 45$ $\min$.

The utilization of diphenylchlorophosphate $\left((\mathrm{PhO})_{2} \mathrm{POCl}\right)$ is able to determine the nature of the chain-end group. When adding excess $(\mathrm{PhO})_{2} \mathrm{POCl}$ to a polymer capped with a carboxylate chain end, a mixed anhydride rearranged to tetraphenoxydiphosphate is formed with a characteristic peak at $-26 \mathrm{ppm}$ in the ${ }^{31} \mathrm{P}$ NMR spectrum. Whereas a 
phosphate will be generated with a peak at $-13 \mathrm{ppm}$ in the presence of alkoxide chain-end group.

\section{Computation details}

To get a deeper insight into the proposed mechanism, theoretical investigations of the initiation step were performed by using the Gaussian 16 package. ${ }^{4}$ For geometry optimization, we resorted to density functional theory (DFT) and used the B3LYP functional with D3BJ dispersion correction, ${ }^{5}$ coupled with the $6-31 \mathrm{G}(\mathrm{d})$ basis set. ${ }^{6-8}$ All the optimized states were confirmed to be minimal or transition states by vibrational analysis at $298.15 \mathrm{~K}$, and the IRC (intrinsic reaction coordinate) calculations at the same level were carried out for each transition state to verify the right connections with its forward and backward minima. ${ }^{9}$ Single point energy were calculated at M06-2X/6-311+G(d, p) level by a self-consistent reaction field (SCRF) with SMD model $^{10-12}$ and toluene was employed as solvent. To increase computational efficiency, the cation framework of catalyst $\mathbf{3}$ was substituted with trimethyl borane.

Initially, the ring-opening of BBL at the acyl-oxygen bond was computed. As shown, it requires a relative Gibbs free energy of $30.5 \mathrm{kcal} / \mathrm{mol}$ and leads to the formation of IN1 with $11.4 \mathrm{kcal} / \mathrm{mol}$, indicating an unfavorable reaction. Then, the ring-opening of $\mathrm{BBL}$ at the alkyl-oxygen bond was also computed. There are two possible pathways to achieve this reaction, one is the benzoate first dissociates from the $\mathrm{B}$ center, and then attacks the monomer activated by trimethyl boron via TS2 with a 29.7 $\mathrm{kcal} / \mathrm{mol}$ overall barrier; the other is the undissociated benzoate directly attacks the methine of BBL via TS3 with a $34.3 \mathrm{kcal} / \mathrm{mol}$ overall barrier. By contrast, we can figure out that the B moiety plays an important role in activating substrate. Both these two pathways lead to the formation of IN2 with a low relative Gibbs free energy of -11.1 $\mathrm{kcal} / \mathrm{mol}$. Taken together, we could conclude that ring-opening at the alkyl-oxygen bond is favored and the B moiety is of vital importance in the ROP. These results also provide 
further supports to the proposed mechanism in the manuscript.

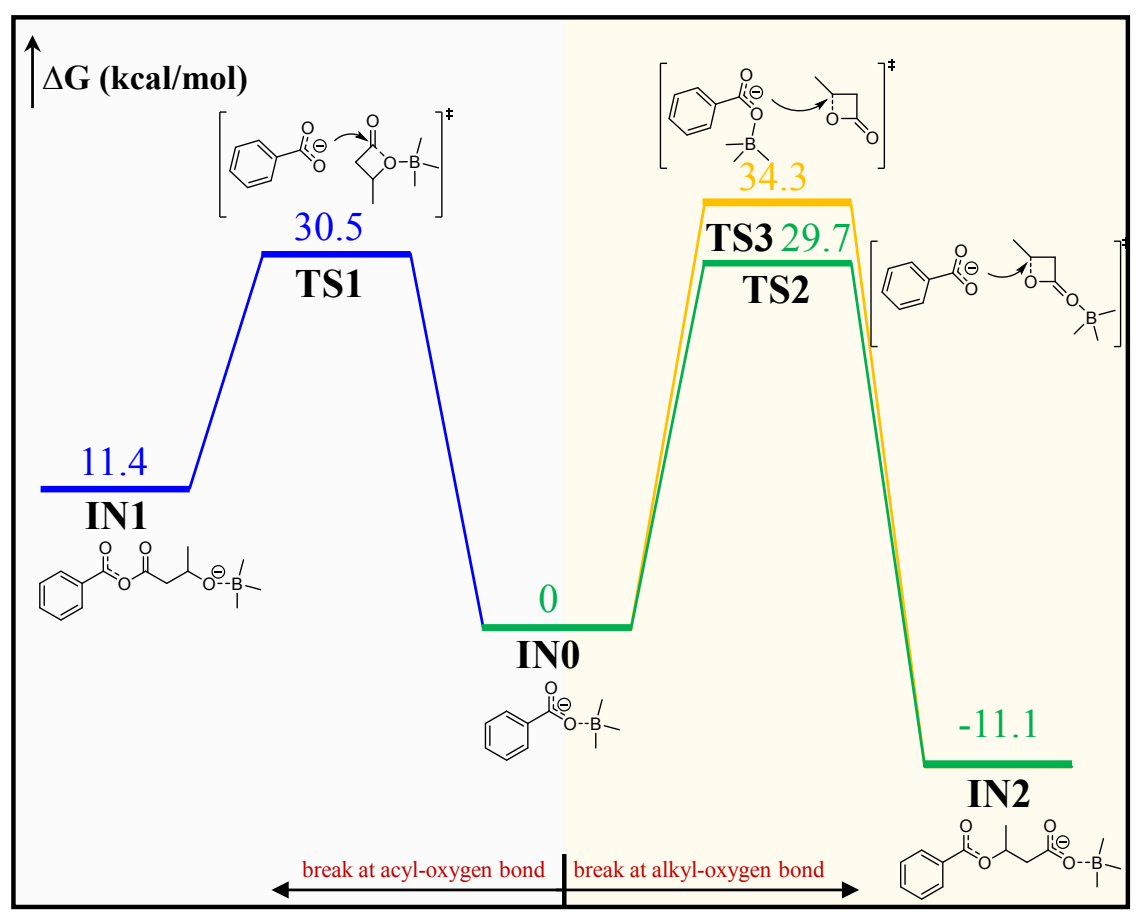

Figure S21. Gibbs free energy profile with the involved intermediates (IN0-IN2) and transition states (TS1-TS3) for the ROP of BBL.

\section{Table S2 for researching side reactions}

Table S2. Prolonging the reaction time for investigation of side reactions in 3-mediated ROP of rac-BBL. ${ }^{a}$

\begin{tabular}{|c|c|c|c|c|c|c|c|c|}
\hline & & rac-BBL & & & \multicolumn{4}{|c|}{ PHB } \\
\hline Entry & Cat. & {$[\mathrm{M}] /[\mathrm{Cat}]$} & $\begin{array}{c}\text { Time }^{b} \\
\text { (h) }\end{array}$ & $\begin{array}{c}\text { Conv }^{c} \\
(\%)\end{array}$ & $\begin{array}{l}\text { Sel. }{ }^{d} \\
(\%)\end{array}$ & $\begin{array}{c}M_{\text {ntheo }}{ }^{e} \\
(\mathrm{~kg} / \mathrm{mol})\end{array}$ & $\begin{array}{c}M_{\mathrm{n} f} \\
(\mathrm{~kg} / \mathrm{mol})\end{array}$ & $\bigoplus^{f}$ \\
\hline 1 & 3 & 100 & 0.5 & 37.2 & $>99$ & 3.2 & 3.1 & 1.08 \\
\hline 2 & 3 & 100 & 1.5 & 98.2 & 92.6 & 7.8 & 5.4 & 1.07 \\
\hline 3 & 3 & 100 & 4.5 & $>99$ & 84.0 & 7.2 & 4.0 & 1.21 \\
\hline 4 & 3 & 100 & 8 & $>99$ & 82.6 & 7.1 & 3.3 & 1.44 \\
\hline 5 & 3 & 100 & 12 & $>99$ & 75.2 & 6.5 & 2.7 & 1.60 \\
\hline
\end{tabular}

${ }^{a}$ All polymerizations run in bulk at $100{ }^{\circ} \mathrm{C}$. ${ }^{b}$ Reaction times were not optimized. ${ }^{c}$ Determined by the integration of ${ }^{1} \mathrm{H}$ NMR methine resonances of BBL, PHB, and crotonic acid. ${ }^{d}$ Determined by the integration of ${ }^{1} \mathrm{H}$ NMR methine resonances of $\mathrm{PHB}$ and crotonic acid. ${ }^{e}$ Calculated from the relation: $[\mathrm{M}] \times$ cat. $^{-1} \times$ conv. $\times$ selectivity $\times M_{\mathrm{BBL}}$ with $M_{\mathrm{BBL}}$ $=86.09 \mathrm{~g} / \mathrm{mol}$ and the mass of end-groups was not involved. ${ }^{~}$ Determined by GPC calibrated with polystyrene standards 


\section{Table S3 for investigating the importance of quaternary ammonium and boron moiety.}

Table S3. Results of control experiments. ${ }^{a}$

\begin{tabular}{|c|c|c|c|c|c|c|c|}
\hline rac-BB & & \multicolumn{2}{|c|}{ PHB } & \multicolumn{2}{|c|}{7} & \multicolumn{2}{|c|}{8} \\
\hline Entry & Cat. & $\mathrm{M} / \mathrm{Cat}$ & $\begin{array}{c}\text { Conv. }^{b} \\
(\%)\end{array}$ & $\begin{array}{l}\text { Sel. } \\
(\%)\end{array}$ & $\begin{array}{c}M_{\text {ntheo }}{ }^{d} \\
(\mathrm{~kg} / \mathrm{mol})\end{array}$ & $\begin{array}{c}M_{\mathrm{n}}{ }^{e} \\
(\mathrm{~kg} / \mathrm{mol})\end{array}$ & $\oplus^{e}$ \\
\hline 1 & 7 & 100 & 0 & - & - & - & - \\
\hline 2 & $\mathrm{PhCOONa}$ & 100 & 7.4 & $>99$ & - & - & - \\
\hline 3 & 8 & 100 & $>99$ & 95.0 & 8.6 & 2.4 & 1.72 \\
\hline 4 & $7 / 8$ & 100 & 93.5 & 94.3 & 8.0 & 3.9 & 1.28 \\
\hline
\end{tabular}

${ }^{a}$ All polymerizations run in bulk at $100{ }^{\circ} \mathrm{C} .{ }^{b}$ Determined by the integration of ${ }^{1} \mathrm{H}$ NMR methine resonances of $r a c$-BBL, $\mathrm{PHB}$, and crotonic acid. ${ }^{c}$ Selectivity of polymer expressed the percentage of polyester in products and was determined by the integration of ${ }^{1} \mathrm{H}$ NMR methine resonances of PHB and crotonic acid. ${ }^{d}$ Calculated from the relation: $[\mathrm{M}] \times$ cat. $^{-1}$ $\times$ conv. $\times$ selectivity $\times M_{\mathrm{BBL}}$ with $M_{\mathrm{BBL}}=86.09 \mathrm{~g} / \mathrm{mol}$ and the mass of end-groups was not involved. ${ }^{e}$ Determined by GPC calibrated with polystyrene standards in THF at $35^{\circ} \mathrm{C}$.

\section{The detailed MALDI-TOF spectrum of PHB in Figure 4A}




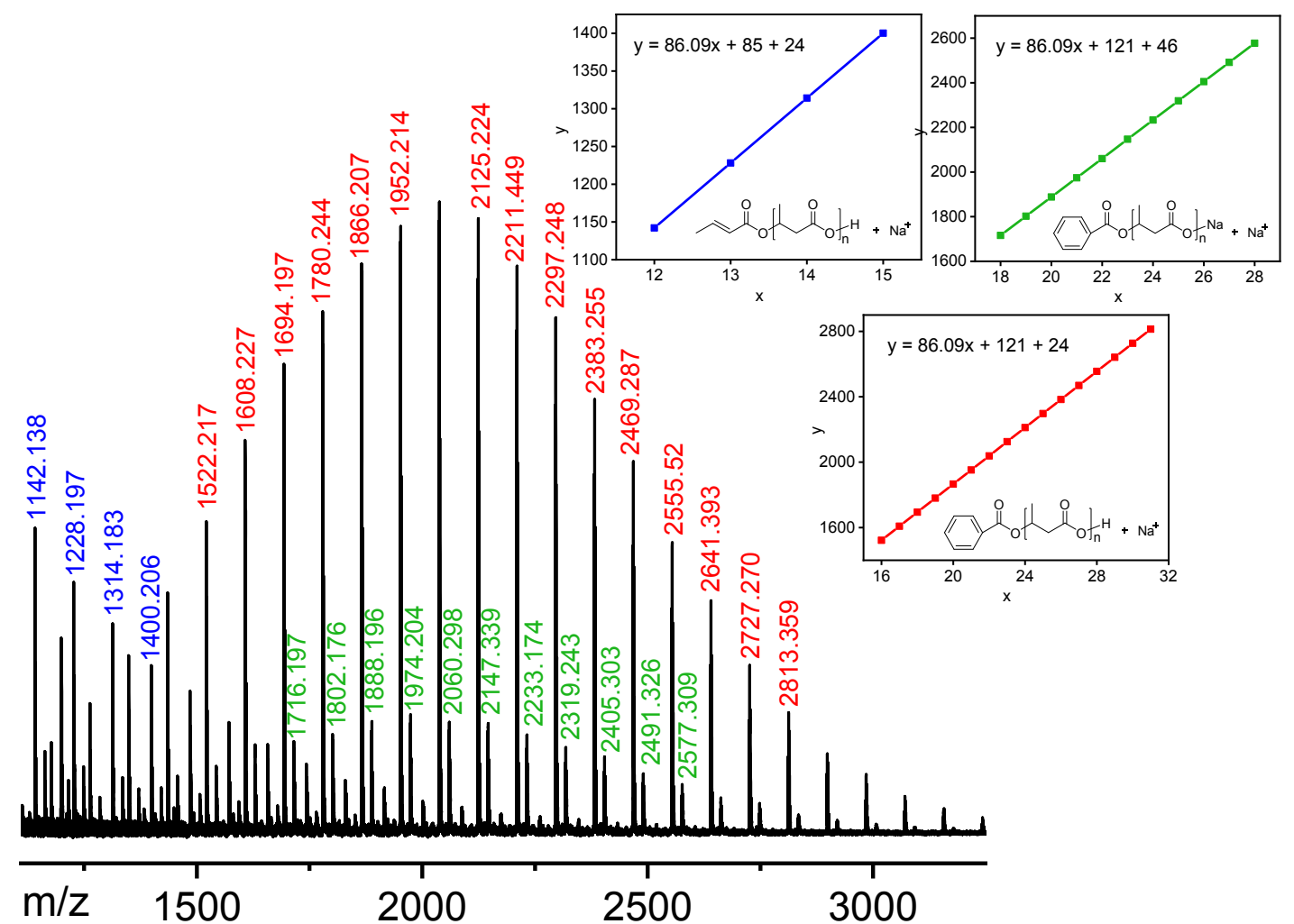

Figure S22. The MALDI-TOF spectrum of PHB produced with $\mathrm{rac}$-BBL/3 $\left(100 / 1,100^{\circ} \mathrm{C}, 0.5 \mathrm{~h}\right)$.

The whole MALDI TOF spectrum of PHB produced in $4.5 \mathrm{~h}$

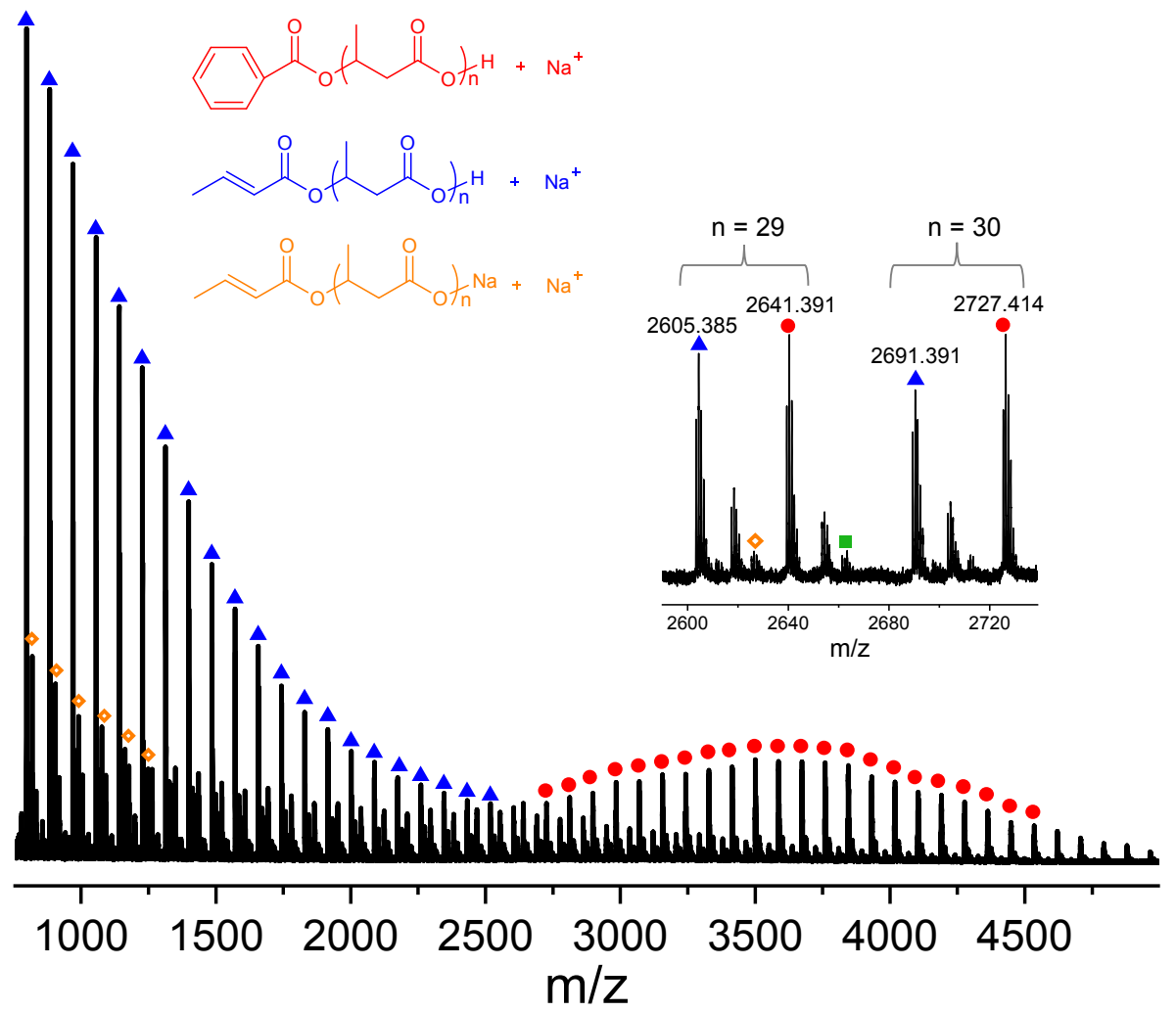


Figure S23. The whole MALDI TOF mass spectrum of the PHB sample by 3. Reaction conditions: rac-BBL/3 $=100 / 1,100{ }^{\circ} \mathrm{C}, 4.5 \mathrm{~h}$. The major population $\left(\left[\left(\mathrm{C}_{4} \mathrm{H}_{6} \mathrm{O}_{2}\right) \mathrm{n}+\mathrm{C}_{4} \mathrm{H}_{5} \mathrm{O}_{2}+\mathrm{H}+\mathrm{Na}\right]^{+}, \mathrm{m} / \mathrm{z}=\right.$ $86.09 \mathrm{n}+85+1+23)$, was marked by blue triangles. The second population $\left(\left[\left(\mathrm{C}_{4} \mathrm{H}_{6} \mathrm{O}_{2}\right) \mathrm{n}+\mathrm{C}_{7} \mathrm{H}_{5} \mathrm{O}_{2}\right.\right.$ $\left.+\mathrm{H}+\mathrm{Na}]^{+}, \mathrm{m} / \mathrm{z}=86.09 \mathrm{n}+121+1+23\right)$, was marked by red dots. The third distribution, marked by orange hollow square, was the counterpart of major population where $\mathrm{H}^{+}$was exchanged with $\mathrm{Na}^{+}$ $\left(\left[\left(\mathrm{C}_{4} \mathrm{H}_{6} \mathrm{O}_{2}\right) \mathrm{n}+\mathrm{C}_{4} \mathrm{H}_{5} \mathrm{O}_{2}+\mathrm{Na}+\mathrm{Na}\right]^{+}, \mathrm{m} / \mathrm{z}=86.09 \mathrm{n}+85+23+23\right)$.

\section{${ }^{1} \mathrm{H}$ and ${ }^{13} \mathrm{C}$ NMR spectra of synthesized compounds}

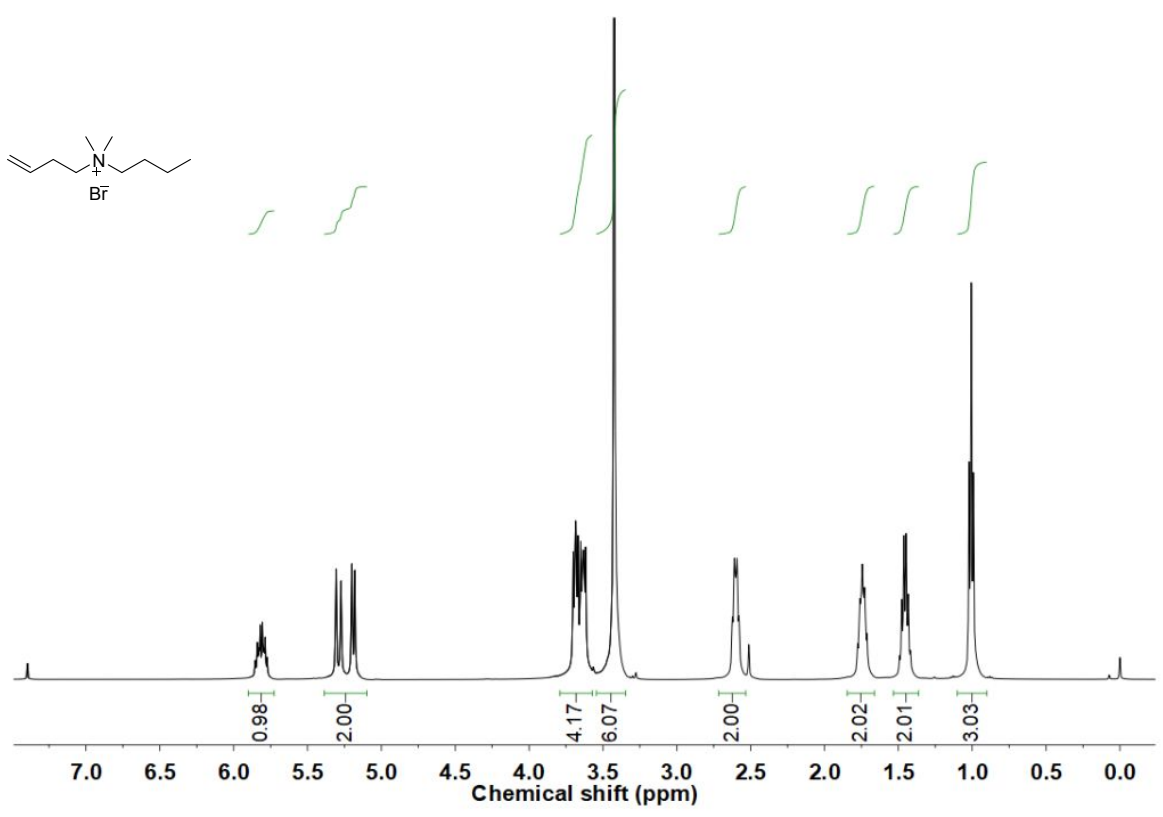



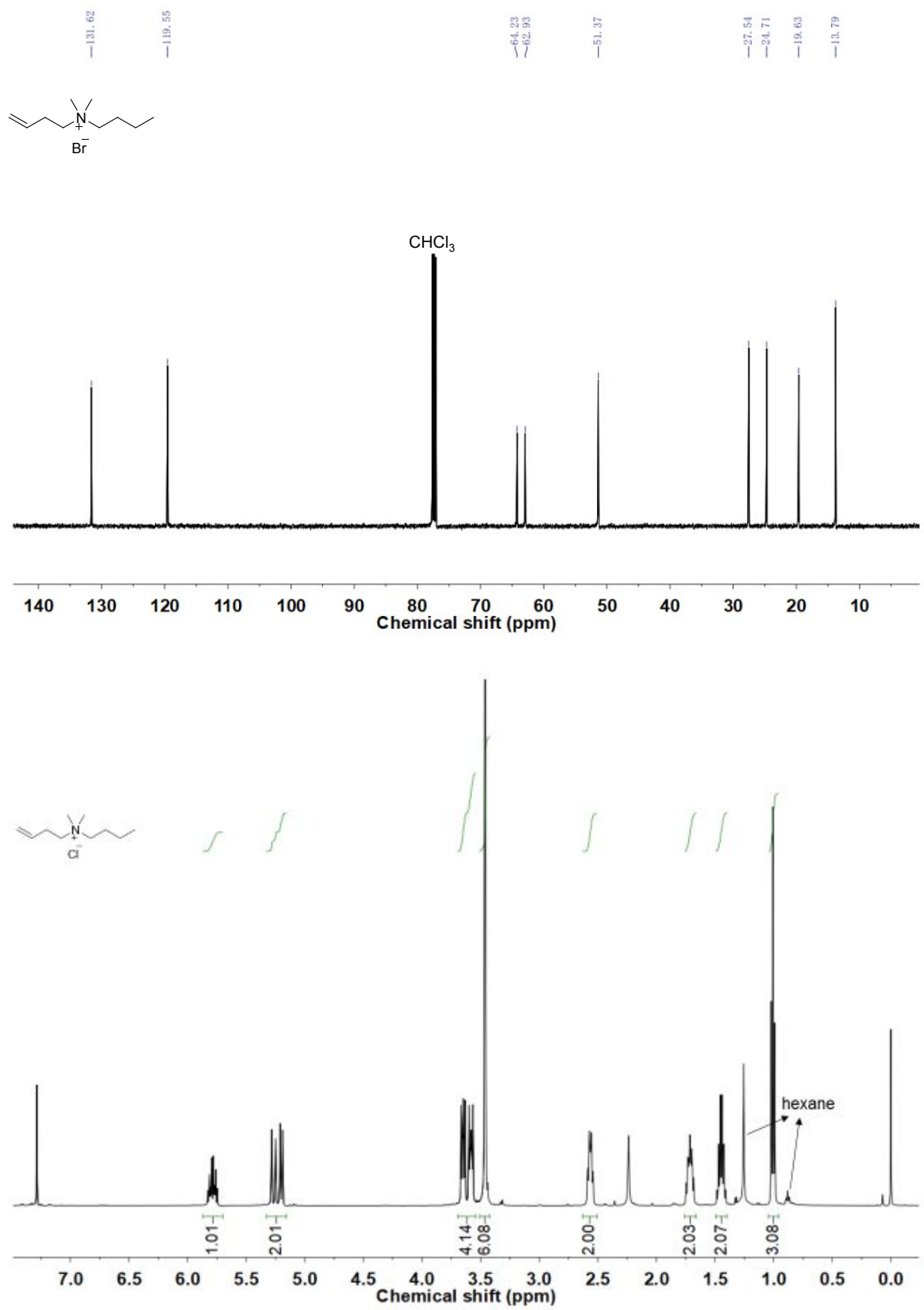

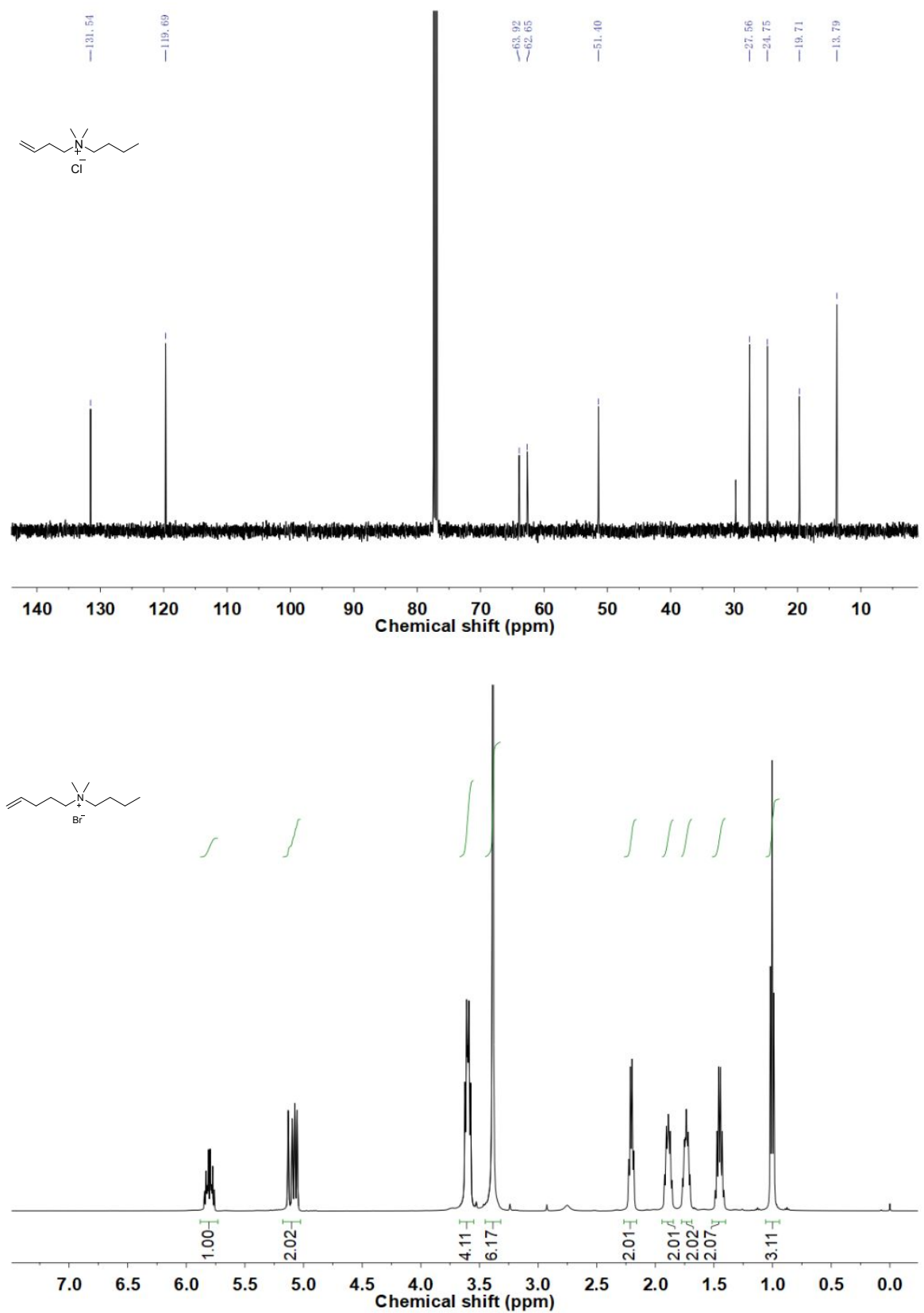

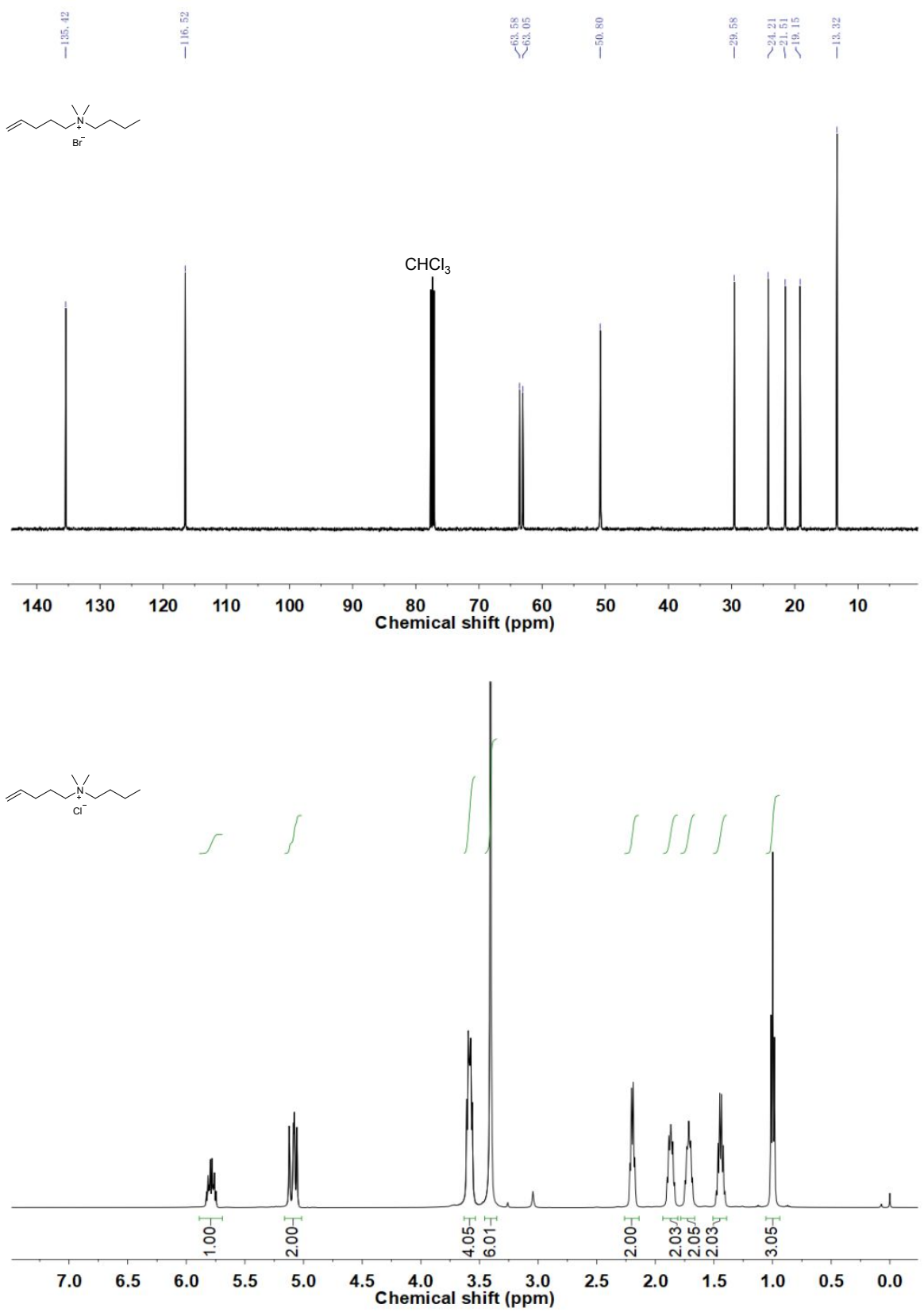

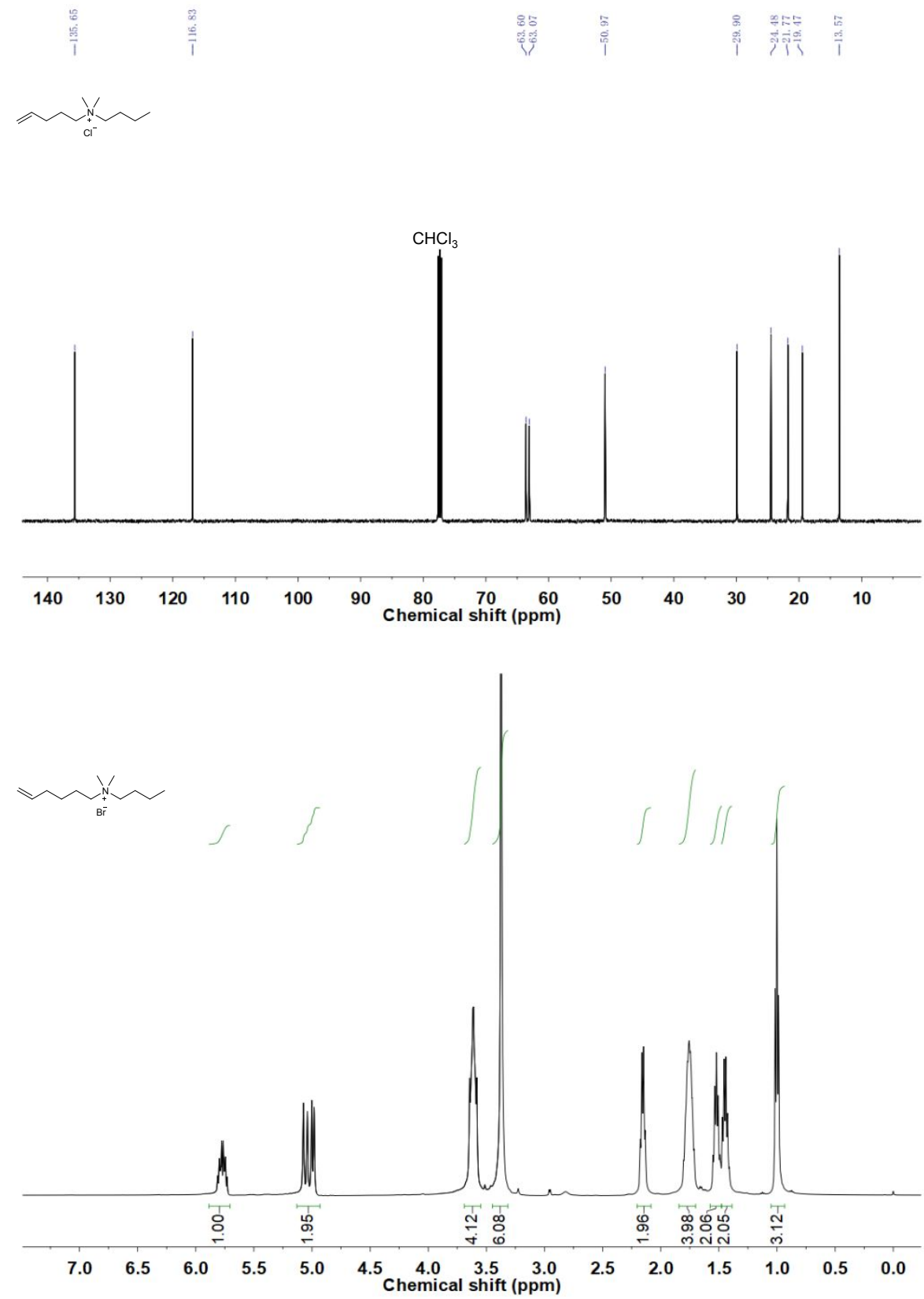


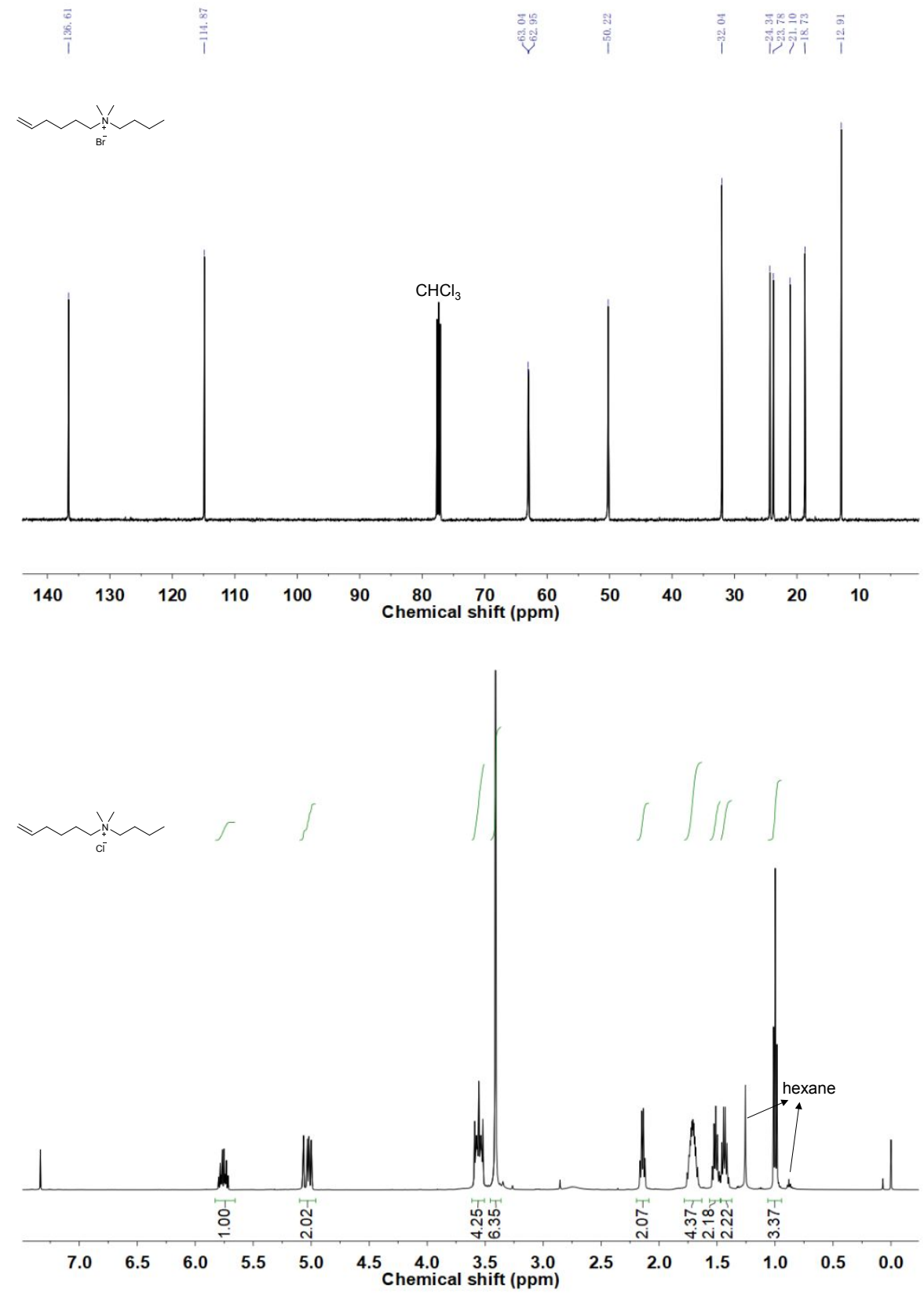



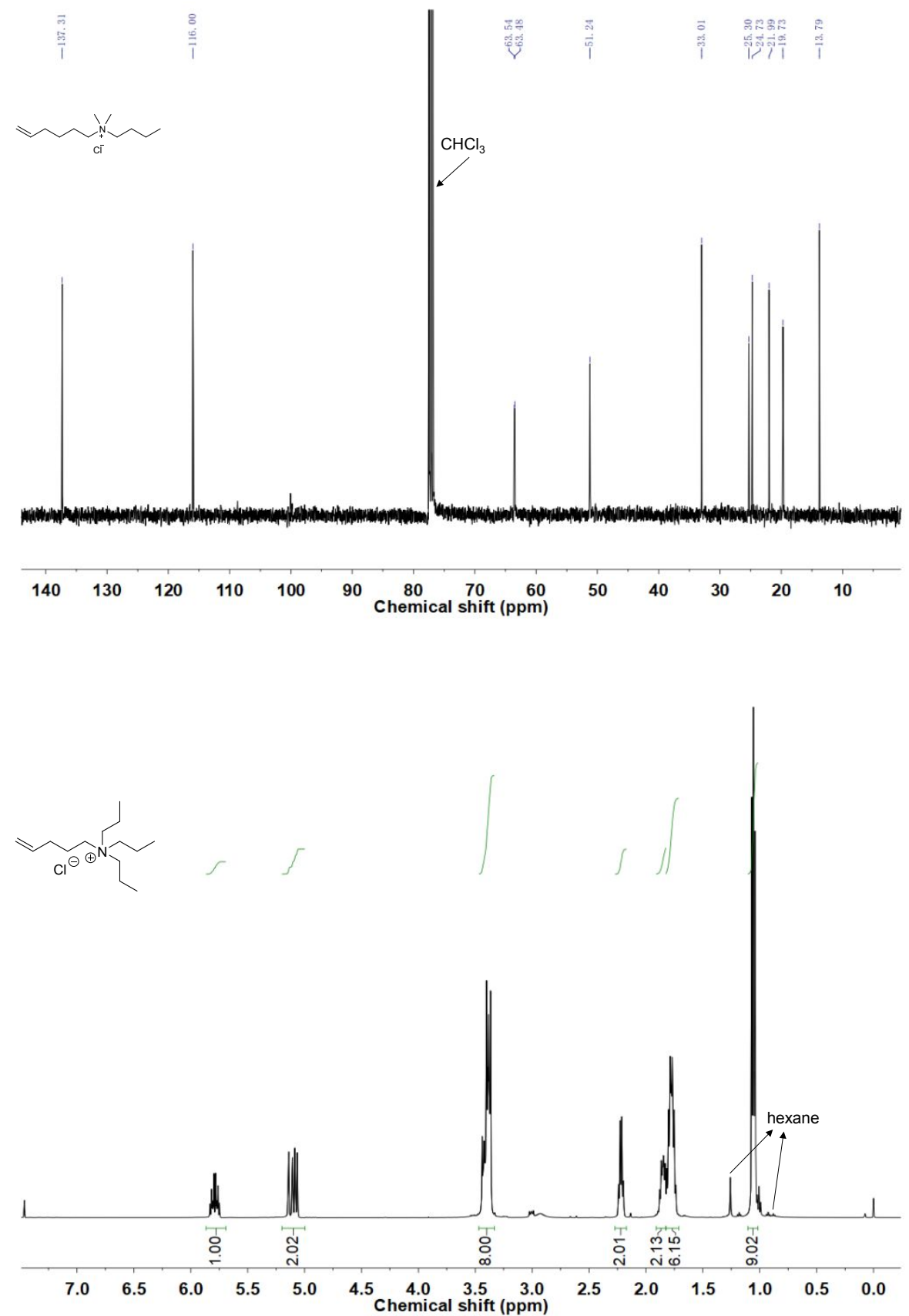

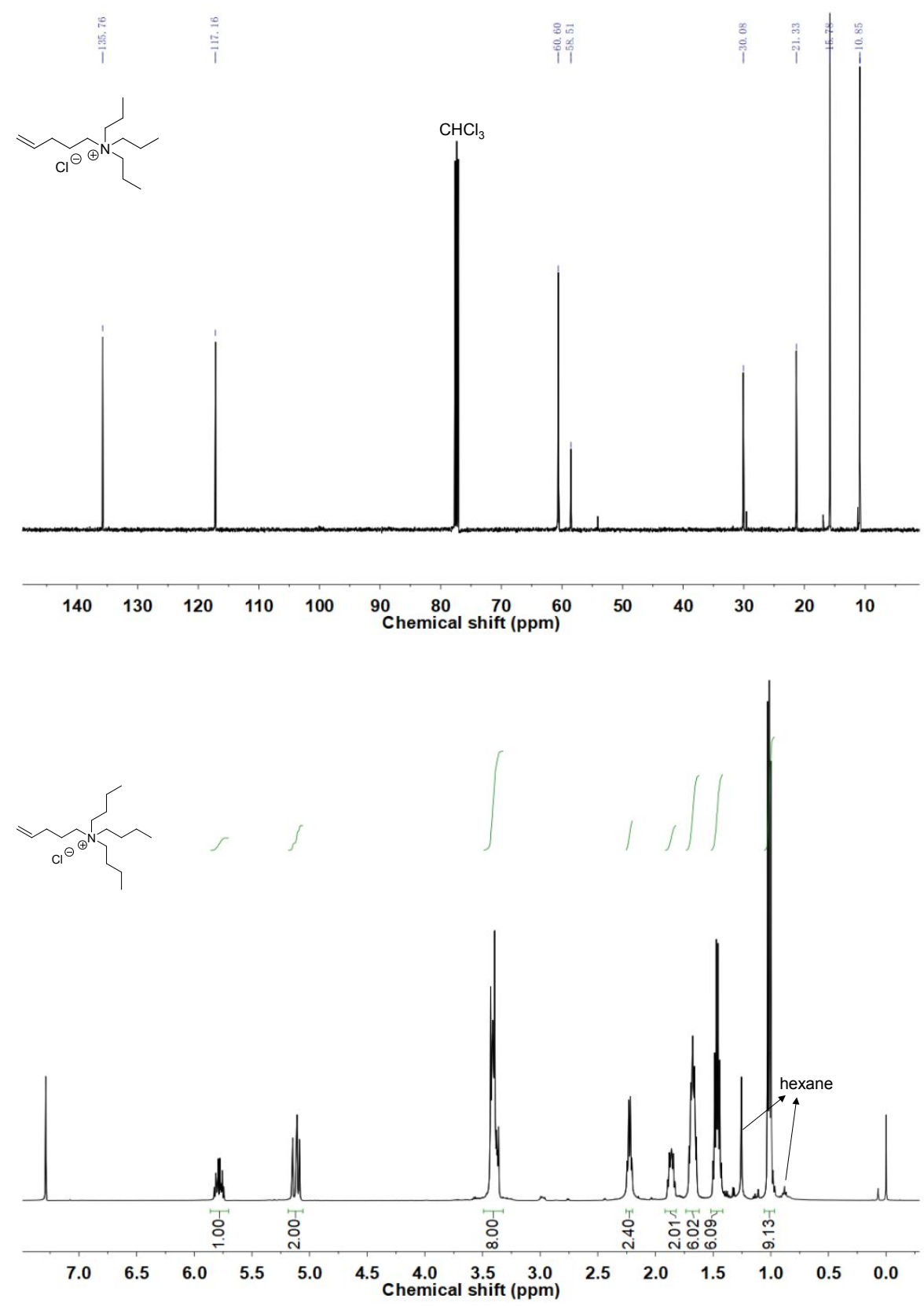


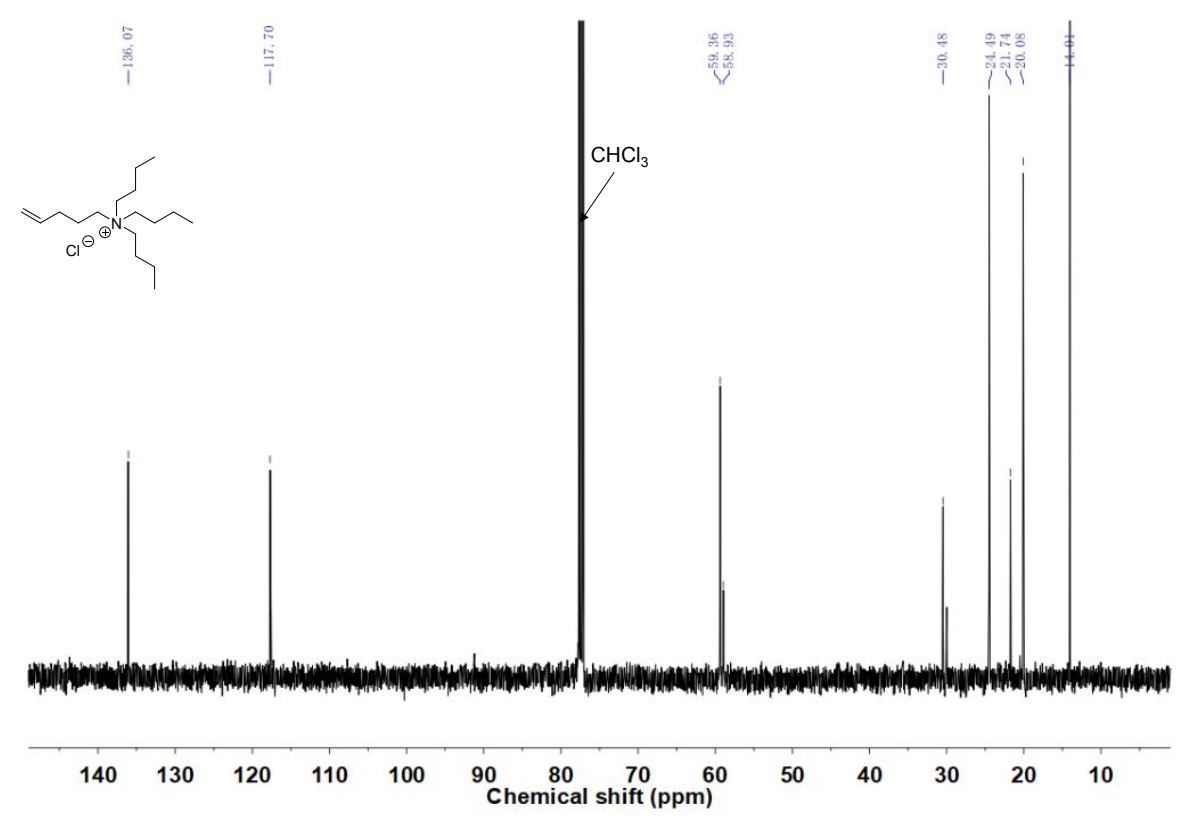

\section{Cartesian coordinates for the optimized structures}

\section{BBL}

$\begin{array}{lrrc}\mathrm{C} & 0.10595500 & 1.16103800 & 0.08522000 \\ \mathrm{C} & -0.90987000 & 0.07186300 & 0.47930900 \\ \mathrm{O} & 0.14124700 & -0.95736700 & 0.31714900 \\ \mathrm{H} & 0.37472600 & 1.88627200 & 0.85681600 \\ \mathrm{H} & -0.09321400 & 1.67932200 & -0.85659200 \\ \mathrm{O} & 2.22414700 & -0.18957400 & -0.31878900 \\ \mathrm{C} & 1.07588100 & -0.01389700 & -0.03859300 \\ \mathrm{C} & -2.08109300 & -0.17966500 & -0.44177100 \\ \mathrm{H} & -2.56252900 & -1.13304000 & -0.20283700 \\ \mathrm{H} & -2.82371800 & 0.61896400 & -0.33341700 \\ \mathrm{H} & -1.75131000 & -0.21091900 & -1.48561900 \\ \mathrm{H} & -1.21234600 & 0.09889400 & 1.52978900\end{array}$

INO

$\begin{array}{lrrc}\mathrm{O} & 1.01317700 & 0.33730200 & -0.00011800 \\ \mathrm{O} & 0.43648700 & -1.86817200 & 0.00008400 \\ \mathrm{C} & 0.18679700 & -0.66163700 & -0.00001600 \\ \mathrm{C} & -1.26471500 & -0.20703000 & -0.00002100 \\ \mathrm{C} & -1.61220400 & 1.14849700 & -0.00000400 \\ \mathrm{C} & -2.27794700 & -1.17094400 & -0.00002300 \\ \mathrm{C} & -2.95341600 & 1.53238200 & 0.00001900 \\ & & \mathrm{~S} 38 & \end{array}$




$\begin{array}{lrrc}\text { C } & -3.61905900 & -0.79030800 & -0.00001800 \\ \text { C } & -3.96109500 & 0.56457300 & 0.00000300 \\ \text { B } & 2.60073900 & 0.24509400 & -0.00001300 \\ \mathrm{H} & -0.81227900 & 1.88062200 & -0.00002100 \\ \mathrm{H} & -1.97760700 & -2.21388900 & -0.00001100 \\ \mathrm{H} & -3.21513600 & 2.58858300 & 0.00006500 \\ \mathrm{H} & -4.40020400 & -1.54798200 & -0.00005900 \\ \mathrm{H} & -5.00712300 & 0.86443700 & 0.00001600 \\ \mathrm{C} & 3.04957300 & 1.81780600 & -0.00002100 \\ \mathrm{H} & 4.14687000 & 1.92140400 & 0.00001100 \\ \mathrm{H} & 2.68096600 & 2.36199200 & -0.88528800 \\ \mathrm{H} & 2.68090900 & 2.36208500 & 0.88516200 \\ \mathrm{C} & 3.06972800 & -0.52581600 & 1.36157200 \\ \mathrm{H} & 4.16929500 & -0.55021400 & 1.44128600 \\ \mathrm{H} & 2.70152100 & -0.02682800 & 2.27353400 \\ \mathrm{H} & 2.71767000 & -1.56531600 & 1.39554500 \\ \mathrm{C} & 3.06985800 & -0.52593500 & -1.36148400 \\ \mathrm{H} & 2.70181400 & -0.02709100 & -2.27358600 \\ \mathrm{H} & 4.16943900 & -0.55041700 & -1.44102900 \\ \mathrm{H} & 2.71774400 & -1.56542600 & -1.39533500\end{array}$

TS1

$\begin{array}{lccc}\mathrm{C} & -1.62888800 & -2.13526200 & -0.22932600 \\ \mathrm{C} & -2.19951100 & -0.96745600 & -1.03911400 \\ \mathrm{O} & -2.29177100 & -0.12070400 & 0.13523700 \\ \mathrm{H} & -0.74136800 & -2.64638200 & -0.59667100 \\ \mathrm{H} & -2.40021000 & -2.85650300 & 0.06742100 \\ \mathrm{O} & -1.36095400 & -1.27129600 & 2.12366800 \\ \mathrm{C} & -1.34687400 & -1.19641100 & 0.93781000 \\ \mathrm{C} & -3.53619800 & -1.17020300 & -1.72435800 \\ \mathrm{H} & -3.89127000 & -0.23781800 & -2.17122000 \\ \mathrm{H} & -3.44092100 & -1.92293500 & -2.51785100 \\ \mathrm{H} & -4.28524100 & -1.51253200 & -1.00201800 \\ \mathrm{H} & -1.45880500 & -0.55060100 & -1.72627400 \\ \mathrm{~B} & -2.40675600 & 1.52214200 & 0.12175400 \\ \mathrm{C} & 2.52265600 & 1.12691200 & 0.03390800 \\ \mathrm{C} & 3.77915500 & -0.92307600 & -0.20341600 \\ \mathrm{C} & 4.94870600 & -0.18909000 & -0.39457600 \\ \mathrm{C} & 4.90759900 & 1.20751500 & -0.37247800 \\ & & & \\ & & 539 & \end{array}$




$\begin{array}{lrrr}\mathrm{C} & 3.69254100 & 1.86241700 & -0.15743800 \\ \mathrm{H} & 3.77652300 & -2.00832800 & -0.21299700 \\ \mathrm{H} & 5.89337200 & -0.70288600 & -0.56075100 \\ \mathrm{H} & 5.81896500 & 1.78280300 & -0.52170400 \\ \mathrm{H} & 3.65664400 & 2.94932900 & -0.13884500 \\ \mathrm{C} & 2.56023900 & -0.27194200 & 0.01089900 \\ \mathrm{C} & 1.30303000 & -1.10253200 & 0.20966000 \\ \mathrm{O} & 1.38886400 & -2.33834900 & 0.20853800 \\ \mathrm{O} & 0.23551700 & -0.38503100 & 0.34284900 \\ \mathrm{H} & 1.56988200 & 1.61679000 & 0.20251000 \\ \mathrm{C} & -4.00838700 & 1.82719100 & 0.05739000 \\ \mathrm{H} & -4.49162800 & 1.53174200 & -0.88738600 \\ \mathrm{H} & -4.55974100 & 1.33131900 & 0.87050800 \\ \mathrm{H} & -4.18867000 & 2.90860700 & 0.16760100 \\ \mathrm{C} & -1.74524500 & 1.98674200 & 1.52627700 \\ \mathrm{H} & -2.25019600 & 1.55210300 & 2.40050700 \\ \mathrm{H} & -0.68561100 & 1.71428800 & 1.60301400 \\ \mathrm{H} & -1.81507400 & 3.08177400 & 1.62708700 \\ \mathrm{C} & -1.59473900 & 2.05367400 & -1.18374000 \\ \mathrm{H} & -0.54817000 & 1.72088800 & -1.18789600 \\ \mathrm{H} & -2.03894500 & 1.75495800 & -2.14822200 \\ \mathrm{H} & -1.58349000 & 3.15484800 & -1.18690400\end{array}$

IN1

$\begin{array}{lccc}\mathrm{C} & -1.46892900 & -2.11303400 & -0.43254000 \\ \mathrm{C} & -1.56103200 & -0.63577500 & -0.88942600 \\ \mathrm{O} & -1.90199800 & 0.11179700 & 0.21968100 \\ \mathrm{H} & -0.95991600 & -2.72547500 & -1.18515500 \\ \mathrm{H} & -2.47392800 & -2.51318200 & -0.27252500 \\ \mathrm{O} & -1.16416300 & -2.83163800 & 1.86111000 \\ \mathrm{C} & -0.75927800 & -2.26402500 & 0.88527600 \\ \mathrm{C} & -2.55202700 & -0.56515400 & -2.06515100 \\ \mathrm{H} & -2.59675300 & 0.44850000 & -2.46520600 \\ \mathrm{H} & -2.25492900 & -1.24984400 & -2.87239500 \\ \mathrm{H} & -3.55602600 & -0.83754100 & -1.71983000 \\ \mathrm{H} & -0.56836100 & -0.33961800 & -1.29053800 \\ \mathrm{~B} & -2.14189300 & 1.63594700 & 0.17242000 \\ \mathrm{C} & 1.69921200 & 0.80968900 & 0.66840200 \\ \mathrm{C} & 3.41829900 & -0.28659100 & -0.65536400 \\ & & & \\ & & 540 & \end{array}$




$\begin{array}{lrrr}\mathrm{C} & 4.17725500 & 0.87909900 & -0.63652000 \\ \mathrm{C} & 3.69586100 & 2.01291300 & 0.02722400 \\ \mathrm{C} & 2.45644300 & 1.97991200 & 0.66762000 \\ \mathrm{H} & 3.75849500 & -1.17573400 & -1.17670500 \\ \mathrm{H} & 5.13864700 & 0.91078200 & -1.14290600 \\ \mathrm{H} & 4.28510700 & 2.92681600 & 0.03232400 \\ \mathrm{H} & 2.06219900 & 2.86709800 & 1.15369400 \\ \mathrm{C} & 2.18606000 & -0.32501600 & 0.00928900 \\ \mathrm{C} & 1.38375400 & -1.57159600 & -0.03378800 \\ \mathrm{O} & 1.53951500 & -2.47591900 & -0.82974000 \\ \mathrm{O} & 0.48451600 & -1.61319200 & 0.98945200 \\ \mathrm{H} & 0.71829500 & 0.77967300 & 1.12850400 \\ \mathrm{C} & -3.73102500 & 1.93693800 & -0.15654000 \\ \mathrm{H} & -4.03960400 & 1.68410600 & -1.18385400 \\ \mathrm{H} & -4.39569200 & 1.37211600 & 0.51742900 \\ \mathrm{H} & -3.97588800 & 3.00374500 & -0.01822500 \\ \mathrm{C} & -1.80523300 & 2.15079400 & 1.69751000 \\ \mathrm{H} & -2.37279100 & 1.58361600 & 2.45204800 \\ \mathrm{H} & -0.74106500 & 2.05346500 & 1.97589900 \\ \mathrm{H} & -2.06221700 & 3.21380600 & 1.83684900 \\ \mathrm{C} & -1.16585500 & 2.39775600 & -0.91754300 \\ \mathrm{H} & -0.09340300 & 2.19712800 & -0.77080800 \\ \mathrm{H} & -1.37865300 & 2.16806400 & -1.97500600 \\ \mathrm{H} & -1.28803400 & 3.48889700 & -0.82042300\end{array}$

TS2

$\begin{array}{lrrr}\mathrm{C} & -1.38990900 & 0.20309800 & 0.18449700 \\ \mathrm{C} & -0.65877600 & 1.53187200 & 0.18650700 \\ \mathrm{O} & -2.35329200 & 2.09149100 & -0.40414400 \\ \mathrm{H} & -1.42482600 & -0.29623300 & 1.15432900 \\ \mathrm{H} & -0.99441200 & -0.49710900 & -0.55128200 \\ \mathrm{H} & -0.08648900 & 1.80450500 & -0.69012900 \\ \mathrm{C} & -0.55000400 & 2.36899000 & 1.42677300 \\ \mathrm{H} & 0.40014200 & 2.15063800 & 1.91683400 \\ \mathrm{H} & -1.37089100 & 2.14983200 & 2.11786800 \\ \mathrm{H} & -0.58859300 & 3.43151800 & 1.16845100 \\ \mathrm{O} & -3.83434200 & 0.41068200 & -0.41971200 \\ \mathrm{C} & -2.67822000 & 0.85899100 & -0.24049900 \\ \mathrm{~B} & -4.37247700 & -1.11352500 & -0.09828700 \\ & & & \end{array}$




$\begin{array}{lrrr}\mathrm{C} & -5.85602600 & -1.08721600 & -0.75544500 \\ \mathrm{H} & -6.37609700 & -2.04164800 & -0.58045900 \\ \mathrm{H} & -6.49092300 & -0.29520200 & -0.33075700 \\ \mathrm{H} & -5.83390500 & -0.93301200 & -1.84497700 \\ \mathrm{C} & -4.39886900 & -1.23651700 & 1.52332300 \\ \mathrm{H} & -4.83877400 & -2.20068200 & 1.82127500 \\ \mathrm{H} & -3.40355300 & -1.19780100 & 1.99276500 \\ \mathrm{H} & -5.00953900 & -0.45237600 & 1.99568200 \\ \mathrm{C} & -3.38557100 & -2.17918600 & -0.82999900 \\ \mathrm{H} & -2.40520900 & -2.31052900 & -0.35008600 \\ \mathrm{H} & -3.85984600 & -3.17257400 & -0.82512900 \\ \mathrm{H} & -3.20126200 & -1.93092700 & -1.88644200 \\ \mathrm{C} & 3.70361700 & -0.76254000 & 1.03024100 \\ \mathrm{C} & 4.38142600 & 0.03374800 & -1.14360500 \\ \mathrm{C} & 5.63513400 & -0.55141200 & -0.97019400 \\ \mathrm{C} & 5.92637800 & -1.24535700 & 0.20730800 \\ \mathrm{C} & 4.95649800 & -1.34972400 & 1.20751100 \\ \mathrm{H} & 4.12290500 & 0.57846700 & -2.04590500 \\ \mathrm{H} & 6.38754900 & -0.46887000 & -1.75193100 \\ \mathrm{H} & 6.90374700 & -1.70332400 & 0.34418700 \\ \mathrm{H} & 5.17884300 & -1.89053900 & 2.12513900 \\ \mathrm{C} & 3.40662100 & -0.06645100 & -0.14591300 \\ \mathrm{C} & 2.04173700 & 0.58301200 & -0.35293400 \\ \mathrm{O} & 1.84084800 & 1.18536000 & -1.42724100 \\ \mathrm{O} & 1.22394800 & 0.44558400 & 0.62067500 \\ \mathrm{H} & 2.93200000 & -0.82928100 & 1.78995700\end{array}$

\section{TS3}

$\begin{array}{lrrr}\mathrm{C} & -3.03052600 & -1.37533100 & -0.17500600 \\ \mathrm{C} & -2.34099800 & -0.16268700 & 0.37809300 \\ \mathrm{O} & -4.36141400 & 0.46409500 & -0.03751900 \\ \mathrm{H} & -3.02086100 & -2.21600400 & 0.52701800 \\ \mathrm{H} & -2.57990400 & -1.71248500 & -1.11341300 \\ \mathrm{H} & -2.03454000 & 0.61684300 & -0.30154700 \\ \mathrm{C} & -2.33711900 & 0.14120300 & 1.84898700 \\ \mathrm{H} & -1.60663500 & 0.92128200 & 2.07267500 \\ \mathrm{H} & -2.08642200 & -0.76038500 & 2.41783600 \\ \mathrm{H} & -3.33398200 & 0.47909600 & 2.13824200 \\ \mathrm{O} & -5.40776400 & -1.41574400 & -0.78870600 \\ & & & \\ & & \mathrm{~S} 42 & \end{array}$




$\begin{array}{lrrc}\mathrm{C} & -4.44192300 & -0.77524800 & -0.37873600 \\ \mathrm{~B} & 1.05468600 & 2.39600900 & -0.37077400 \\ \mathrm{C} & -0.27826000 & 3.15787600 & -0.89790100 \\ \mathrm{H} & -0.01868500 & 4.11590600 & -1.37513100 \\ \mathrm{H} & -0.83201100 & 2.57329000 & -1.64815500 \\ \mathrm{H} & -0.98286000 & 3.38586300 & -0.08524800 \\ \mathrm{C} & 2.01011600 & 1.91302400 & -1.59152600 \\ \mathrm{H} & 2.23914700 & 2.78926100 & -2.21758500 \\ \mathrm{H} & 2.97533000 & 1.48071100 & -1.29724700 \\ \mathrm{H} & 1.52021900 & 1.18274900 & -2.25395200 \\ \mathrm{C} & 1.81153600 & 3.21479800 & 0.81001700 \\ \mathrm{H} & 2.73162500 & 2.74074700 & 1.18725000 \\ \mathrm{H} & 2.11373700 & 4.20597800 & 0.43825800 \\ \mathrm{H} & 1.15978500 & 3.39100800 & 1.67828400 \\ \mathrm{C} & 1.88422800 & -2.12341200 & -0.48701300 \\ \mathrm{C} & 2.96410200 & -0.27812900 & 0.64268400 \\ \mathrm{C} & 4.18661400 & -0.94177200 & 0.55299800 \\ \mathrm{C} & 4.26374700 & -2.18735900 & -0.07249200 \\ \mathrm{C} & 3.10977800 & -2.77637100 & -0.59539200 \\ \mathrm{H} & 2.89304600 & 0.69407100 & 1.11405400 \\ \mathrm{H} & 5.08096800 & -0.48234900 & 0.96585200 \\ \mathrm{H} & 5.21975700 & -2.69955800 & -0.15061500 \\ \mathrm{H} & 3.16556300 & -3.74692100 & -1.08184400 \\ \mathrm{C} & 1.80786600 & -0.86406000 & 0.11930600 \\ \mathrm{C} & 0.47314200 & -0.19302600 & 0.26777800 \\ \mathrm{O} & 0.36996500 & 1.06792800 & 0.37440400 \\ \mathrm{O} & -0.51874100 & -0.97415200 & 0.34864800 \\ \mathrm{H} & 0.97311200 & -2.57319300 & -0.86625900\end{array}$

\section{IN2}

C

C

$\mathrm{O}$

$\mathrm{H}$

$\mathrm{H}$

$\mathrm{H}$

C

$\mathrm{H}$

$\mathrm{H}$
0.83468400

0.26994500

2.87646400

0.51862500

0.42874700

0.50430800

0.71906800

0.23716000

0.46630000
0.38248100

0.47122200

$-0.19384500$

1.24973900

$-0.51204000$

$-0.44123300$

1.69961500

1.73823900

2.61498000
$-1.03030000$

0.38247600

0.00525400

$-1.62027300$

$-1.51819100$

0.92982900

1.15302800

2.13614200

0.60442200 


$\begin{array}{lrrr}\mathrm{H} & 1.80141900 & 1.65030100 & 1.28684800 \\ \mathrm{O} & 2.95406000 & 0.65582800 & -2.10977900 \\ \mathrm{C} & 2.37589000 & 0.29536500 & -1.08976500 \\ \mathrm{~B} & 4.42587700 & -0.36169500 & 0.33889000 \\ \mathrm{C} & 5.11165900 & 1.12066100 & 0.29120500 \\ \mathrm{H} & 6.17403100 & 1.07398200 & 0.58228700 \\ \mathrm{H} & 5.06822100 & 1.56980900 & -0.70987500 \\ \mathrm{H} & 4.63171400 & 1.83005400 & 0.98778400 \\ \mathrm{C} & 5.07962400 & -1.40295800 & -0.73390800 \\ \mathrm{H} & 6.13678100 & -1.60538300 & -0.49410800 \\ \mathrm{H} & 4.56726600 & -2.37928600 & -0.73434200 \\ \mathrm{H} & 5.04608000 & -1.01818500 & -1.76169500 \\ \mathrm{C} & 4.39654800 & -0.97399500 & 1.85457300 \\ \mathrm{H} & 3.86777800 & -1.93941600 & 1.90917900 \\ \mathrm{H} & 5.41577900 & -1.14884900 & 2.23564300 \\ \mathrm{H} & 3.90262400 & -0.30005200 & 2.57467800 \\ \mathrm{C} & -3.89656000 & 1.03812600 & 0.09918500 \\ \mathrm{C} & -4.25101800 & -1.35269300 & -0.03795400 \\ \mathrm{C} & -5.62497700 & -1.14534900 & -0.13023300 \\ \mathrm{C} & -6.13798200 & 0.15389600 & -0.10850100 \\ \mathrm{C} & -5.27213500 & 1.24361200 & 0.00579400 \\ \mathrm{H} & -3.82408400 & -2.34983800 & -0.05368600 \\ \mathrm{H} & -6.29688200 & -1.99485900 & -0.22011000 \\ \mathrm{H} & -7.21037800 & 0.31678800 & -0.18138000 \\ \mathrm{H} & -5.66945000 & 2.25508900 & 0.02154200 \\ \mathrm{C} & -3.38073900 & -0.26308300 & 0.07782700 \\ \mathrm{O} & -1.91130700 & -0.54294700 & 0.17381600 \\ \mathrm{H} & -1.45037500 & -1.67002300 & 0.14192300 \\ & -1.19999900 & 0.58060800 & 0.30015000 \\ \mathrm{H} & -31282000 & 1.87436800 & 0.18702100\end{array}$




\section{Reference}

1. Yang, G.-W.; Zhang, Y.-Y.; Xie, R.; Wu, G.-P. Scalable Bifunctional Organoboron Catalysts for Copolymerization of $\mathrm{CO}_{2}$ and Epoxides with Unprecedented Efficiency. $\mathrm{J}$. Am. Chem. Soc. 2020, 142, 12245-12255.

2. Yang, G.-W.; Zhang, Y.-Y.; Xie, R.; Wu, G.-P. High-Activity Organocatalysts for Polyether Synthesis via Intramolecular Ammonium Cation Assisted $\mathrm{S}_{\mathrm{N}} 2$ Ring-Opening Polymerization. Angew. Chem., Int. Ed. 2020, 132, 17058-17065.

3. Zhang, Y.-Y.; Yang, G.-W.; Xie, R.; Yang, L.; Li, B.; Wu, G.-P. Scalable, Durable, and Recyclable Metal-Free Catalysts for Highly Efficient Conversion of $\mathrm{CO}_{2}$ to Cyclic Carbonates. Angew. Chem., Int. Ed. 2020, 59, 23291-23298.

4. M. J. Frisch, G. W. Trucks, H. B. Schlegel, G. E. Scuseria, M. A. Robb, J. R. Cheeseman, G. Scalmani, V. Barone, G. A. Petersson, H. Nakatsuji, X. Li, M. Caricato, A. V. Marenich, J. Bloino, B. G. Janesko, R. Gomperts, B. Mennucci, H. P. Hratchian, J. V. Ortiz, A. F. Izmaylov, J. L. Sonnenberg, D. Williams-Young, F. Ding, F. Lipparini, F. Egidi, J. Goings, B. Peng, A. Petrone, T. Henderson, D. Ranasinghe, V. G. Zakrzewski, J. Gao, N. Rega, G. Zheng, W. Liang, M. Hada, M. Ehara, K. Toyota, R. Fukuda, J. Hasegawa, M. Ishida, T. Nakajima, Y. Honda, O. Kitao, H. Nakai, T. Vreven, K. Throssell, J. A. Montgomery, Jr., J. E. Peralta, F. Ogliaro, M. J. Bearpark, J. J. Heyd, E. N. Brothers, K. N. Kudin, V. N. Staroverov, T. A. Keith, R. Kobayashi, J. Normand, K. Raghavachari, A. P. Rendell, J. C. Burant, S. S. Iyengar, J. Tomasi, M. Cossi, J. M. Millam, M. Klene, C. Adamo, R. Cammi, J. W. Ochterski, R. L. Martin, K. Morokuma, O. Farkas, J. B. Foresman, D. J. Fox, Gaussian 16, Revision A. 03; Gaussian Inc.: Wallingford, CT, 2016.

5. Grimme, S.; Antony, J.; Ehrlich, S.; Krieg, H. A consistent and accurate ab initio parametrization of density functional dispersion correction (DFT-D) for the 94 elements H-Pu. $J$. Chem. Phys. 2010, 132, 154104.

6. Davidson, E. R.; Feller, D. Basis set selection for molecular calculations. Chem. Rev. 1986, 86, 681-696.

7. Lee, C.; Yang, W.; Parr, R. G. Development of the Colle-Salvetti correlation-energy formula into a functional of the electron density. Phys. Rev. B 1988, 37, 785-789.

8. Becke, A. D. Density-functional thermochemistry. III. The role of exact exchange. J. Chem. Phys. 1993, 98, 5648-5652.

9. Fukui, K. The path of chemical reactions - the IRC approach. Acc. Chem. Res. 1981, 14, 363-368.

10. Miertuš, S.; Scrocco, E.; Tomasi, J. Electrostatic interaction of a solute with a continuum. A direct utilizaion of $\mathrm{AB}$ initio molecular potentials for the prevision of solvent effects. Chem. Phys. 1981, 55, 117-129.

11. Tomasi, J.; Persico, M. Molecular Interactions in Solution: An Overview of Methods Based on Continuous Distributions of the Solvent. Chem. Rev. 1994, 94, 2027-2094. 
12. Cossi, M.; Barone, V.; Cammi, R.; Tomasi, J. Ab initio study of solvated molecules: a new implementation of the polarizable continuum model. Chem. Phys. Lett. 1996, 255, 327-335. 